\title{
Uniform distribution of dislocations in Peierls-Nabarro models for semi-coherent interfaces
}

\author{
Silvio Fanzon ${ }^{1} \cdot$ Marcello Ponsiglione ${ }^{2} \cdot$ Riccardo Scala $^{3}$
}

Received: 21 October 2019 / Accepted: 31 May 2020 / Published online: 9 August 2020

(c) The Author(s) 2020

\begin{abstract}
In this paper we introduce Peierls-Nabarro type models for edge dislocations at semi-coherent interfaces between two heterogeneous crystals, and prove the optimality of uniformly distributed edge dislocations. Specifically, we show that the elastic energy $\Gamma$-converges to a limit functional comprised of two contributions: one is given by a constant $c_{\infty}>0$ gauging the minimal energy induced by dislocations at the interface, and corresponding to a uniform distribution of edge dislocations; the other one accounts for the far field elastic energy induced by the presence of further, possibly not uniformly distributed, dislocations. After assuming periodic boundary conditions and formally considering the limit from semi-coherent to coherent interfaces, we show that $c_{\infty}$ is reached when dislocations are evenly-spaced on the one dimensional circle.
\end{abstract}

Mathematics Subject Classification 74N05 $\cdot 74$ N15 $\cdot 49 \mathrm{~J} 45$

Communicated by J.Ball.

Silvio Fanzon

Silvio.Fanzon@uni-graz.at

Marcello Ponsiglione

ponsigli@mat.uniroma1.it

Riccardo Scala

riccardo.scala@unisi.it

1 Institute of Mathematics and Scientific Computing, University of Graz, Heinrichstraße 36, 8010

Graz, Austria

2 Dipartimento di Matematica "G. Castelnuovo", Sapienza Università di Roma, Piazzale A. Moro 2, 00185 Rome, Italy

3 Dipartimento di Ingegneria dell'Informazione e Scienze Matematiche, San Niccolò, via Roma 56, 53100 Siena, Italy 


\section{Contents}

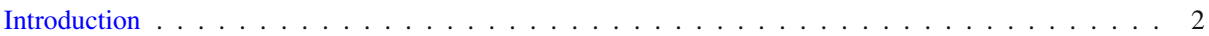

1 Heuristic derivation and related models . . . . . . . . . . . . . . . . . . . . 4

1.1 Semi-coherent interface . . . . . . . . . . . . . . . . . . . . 5

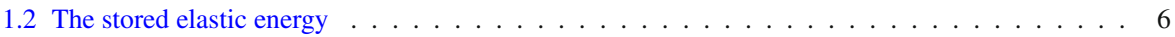

1.3 Comparison with Peierls-Nabarro models . . . . . . . . . . . . . . . . . . . . . . 6

1.4 Comparison with the Ohta-Kawasaki model . . . . . . . . . . . . . . . . . . . 7

2 The mathematical model . . . . . . . . . . . . . . . . . . . . . . . . . . 8

2.1 Admissible configurations and their energy . . . . . . . . . . . . . . . . . 8

2.2 Asymptotic behavior of the energy functionals . . . . . . . . . . . . . . . . . 10

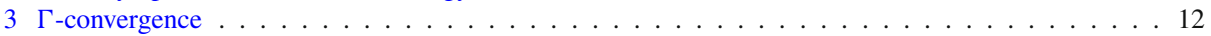

3.1 Proof of $\Gamma$-liminf inequality . . . . . . . . . . . . . . . . . . . . 12

3.2 Proof of the $\Gamma$-limsup inequality . . . . . . . . . . . . . . . . . . . . . 14

4 Periodicity of dislocations on $\mathcal{S}^{1} \ldots \ldots \ldots \ldots \ldots \ldots \ldots$

4.1 Admissible configurations and the energy functional . . . . . . . . . . . . . . . . . 18

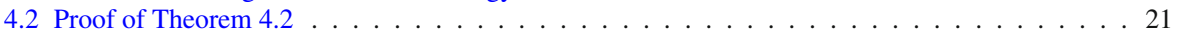

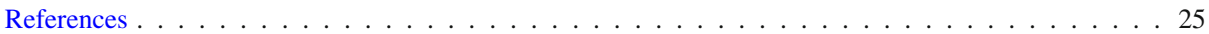

\section{Introduction}

In this paper we provide a rigorous derivation of the uniform distribution of dislocations at flat interface boundaries between heterogeneous two-dimensional crystals whose atomic lattice spacings differ slightly. Such configurations are referred to as semi-coherent interfaces. To this purpose, we propose a variational model based on the classical Peierls-Nabarro and Van der Merwe models [22,26,28]. Our approach consists in minimizing the $H^{\frac{1}{2}}$ seminorm

$$
\int_{0}^{l} \int_{0}^{l} \frac{|u(x)-u(y)|^{2}}{|x-y|^{2}} d x d y,
$$

among admissible displacements $u \in W^{1, \infty}(0, l)$, where $l>0$ represents the size of the interface. In this simplified model, the classical Peierls-Nabarro sinusoidal potential is removed from the energy and replaced by suitable admissibility conditions on the function $u$ : loosely speaking, $u^{\prime}$ can assume only two values (determined by the lattice misfit) on intervals whose size is proportional to the lattice spacing.

It is well known that atomic mismatches are accommodated by periodic array of dislocations, whose presence decreases the energy of the system. This is common of most of the interface boundaries such as small angle grain boundaries, tilt and twist boundaries [27,28]. This is why much effort has been spent in computing the elastic energy induced by periodic distribution of dislocations; we refer to the monograph [23, Sec 3.3] for a comprehensive overview. A relevant theoretical question is to understand optimal configurations of dislocations without assuming their periodicity: rigorous proofs that dislocations are favorable with respect to purely elastic deformations for large interfaces, as well as energy scaling properties have been recently faced in a variety of physical systems related to grain boundaries and epitaxial growth, starting from discrete or semi-discrete models of dislocations $[2,3,6,7,9,10,13,15-18,21]$. The goal of this paper is to analyze the simplified version of the Peierls-Nabarro model, based on the minimization of the energy in (1), without assuming any periodicity on $u$.

We now introduce and derive our model in detail; we consider two lattices $C^{+}$and $C^{-}$, separated by the $x$-axis of $\mathbb{R}^{2}$, with $C^{+}$lying on top of $C^{-}$. For simplicity we assume that $C^{-}$ is rigid and in equilibrium, with lattice spacing equals to $\Delta / 2$, while $C^{+}$is spaced with $\delta / 2$, 
where $0<\delta<\Delta$. We consider the case of a semi-coherent interface, which corresponds to $\delta \approx \Delta$. We analyze the equilibrium conditions for $C^{+}$, which amounts to study the interfacial displacement $u: \frac{\delta}{2} \mathbb{Z} \rightarrow \mathbb{R}$, corresponding to the trace of the full strain at the interface. We are interested in a continuous description of this model, hence we consider a suitable affine extension of $u$, imposing that $u^{\prime} \in\{\lambda,-\Lambda\}$ where $0<\lambda \ll \Lambda$. The region where $u^{\prime}=\lambda$ corresponds to a purely elastic deformation, yielding a perfect interfacial match between $C^{-}$ and $C^{+}$. In contrast $u^{\prime}=-\Lambda$ describes a dislocation core, corresponding in the atomistic picture to the presence of an extra line of atoms, i.e., an edge dislocation (see Fig. 1). For a more precise description of the model we refer to Sect. 1. In this paper we assume that the elastic energy is exactly proportional to the $H^{\frac{1}{2}}$ semi-norm of $u$; this is in contrast with the fact that the elastic energy should depend only on the symmetric part of the strain: indeed our choice should be understood as a mere mathematical simplification.

Minimizing (1) among all functions $u$ with $u^{\prime} \in\{\lambda,-\Lambda\}$, but without any constraint on the size of $\left\{u^{\prime}=-\Lambda\right\}$, yields oscillating minimizing sequences converging uniformly to zero and with vanishing energy. However, the underlying atomic structure imposes further restrictions on $u$, namely that the regions where $u^{\prime}=-\Lambda$ have a minimal length, the core radius $\delta>0$, which is proportional to the lattice spacing. Keeping memory of this important microscopic constraint somehow fixes the frequency of oscillations, leading to a well posed minimization problem.

Since we are interested in the asymptotic behavior of (1) as $l \rightarrow+\infty$, we first observe that the minimal energy diverges with order $l$. Therefore, in order to obtain a meaningful $\Gamma$-convergence result for (1), we rescale the energy by $l$ and introduce the rescaled functions $w_{u} \in H^{\frac{1}{2}}(0,1)$, defined by

$$
w_{u}(x):=\frac{u(x l)}{\sqrt{l}} .
$$

The energy (1), scaled by $l$, reads as

$$
\int_{0}^{1} \int_{0}^{1} \frac{|w(x)-w(y)|^{2}}{|x-y|^{2}} d x d y,
$$

completed with the constraints described above, depending on $l$, hidden in the definition of the class of admissible displacements given in (8), (16).

Our main result is Theorem 3.1, which provides the $\Gamma$-limit of the energy functional (2) as $l \rightarrow+\infty$. More precisely, the limit functional coincides with

$$
c_{\infty}+\int_{0}^{1} \int_{0}^{1} \frac{|w(x)-w(y)|^{2}}{|x-y|^{2}} d x d y,
$$

where $c_{\infty}>0$ is a specific constant and $w$ belongs to $H^{\frac{1}{2}}(0,1)$, without further constraints. In other words, the limit energy splits into two finite contributions: The first, namely the constant $c_{\infty}$, accounts for the minimal energy induced by the dislocations, which are needed to accommodate the interfacial lattice mismatch. Such dislocations are infinitely many and homogeneously distributed on the interval $(0,1)$ (see Theorem 2.6). Indeed, the specific value of $c_{\infty}$ is obtained as the limit as $l \rightarrow+\infty$ of constants $c_{l}$ defined by

$$
c_{l}:=\min _{u} \frac{1}{l} \int_{0}^{l} \int_{0}^{l} \frac{|u(x)-u(y)|^{2}}{|x-y|^{2}} d x d y,
$$


where $u$ is subjected to the usual constraints (see Theorem 2.3). The second term in (3) is induced by the possible presence of further, possibly non uniformly distributed dislocations, inducing a far macroscopic strain.

It would be desirable to compute the constant $c_{l}$ for fixed $l$. However this seems to be out of reach, in particular due to boundary effects at the endpoints of the interval $(0, l)$, which prevent periodic configurations of dislocations. These boundary effects become negligible as $l \rightarrow+\infty$; indeed in Theorem 2.6 we show that, for minimizers, the density of dislocations becomes uniform as $l \rightarrow+\infty$. A further step, which at the present is still missing, would consist in proving real periodicity of the dislocations (in the limit as $l \rightarrow+\infty$ ), and therefore that the constant $c_{\infty}$ agrees with the surface energy density computed in [28].

Finally, we have evidence of the periodic distribution of dislocations for an even more simplified setting, where we assume periodic boundary conditions, fix the number of dislocations, and send $\Lambda \rightarrow+\infty$. Roughly speaking, this process corresponds to consider the limit from semi-coherent to coherent interfaces. More precisely, in Sect. 4 we fix $l$ (and thus the number of dislocations) and we recast our functional (1) as defined on $\mathcal{S}^{1}$ instead of $(0, l)$, thus neglecting the boundary effects. Enforcing the constant $\Lambda \rightarrow+\infty$ and introducing a fixed core-radius $\rho>0$, we show that the optimal positioning of the dislocations is exactly given by evenly-spaced points on the circle $\mathcal{S}^{1}$. This result is proved in Theorem 4.2. The elastic energy given by such a configuration corresponds, in this modified setting, to the limit of $c_{l}$ as $l \rightarrow+\infty$ and $\frac{\delta}{\Delta} \rightarrow 0$ simultaneously and in order to keep the number of dislocations bounded.

From a purely mathematical perspective, let us mention that the understanding of periodic configurations as symmetry breaking minimizers of non convex energy functionals is a fascinating and very active research field. A significant impulse to this subject was given by the work in [20]. Subsequently, much effort has been devoted in seeking periodic minimizers of Ohta-Kawasaki energy functionals [24]. To some extent, our energy can be regarded as a variant of Ohta-Kawasaki energy-type functionals where the $H^{-1}$ norm is replaced by the $H^{-\frac{1}{2}}$ norm [see (7)], and such a variant was already mentioned as relevant in many respects in [20].

The energy in (2) could also be seen as a Modica-Mortola type functional where the Dirichlet term is replaced by the $H^{\frac{1}{2}}$ seminorm $[1,5,8,11,12,14,25]$.

Here the main difference is that our energy functional formally corresponds to a fractional Modica-Mortola type energy, keeping into account a pre-existing strain, which naturally arises as a consequence of the interfacial lattice mismatch, see (6).

While in this paper we enforce the scale of the oscillations by imposing suitable constraints on the class of admissible displacements, in the spirit of the so called core radius approaches, it would be interesting to study relaxed energies where the length-scale is coerced through scale parameters whose purpose is to tune penalizing potentials; such an approach is more closely related to classical Ohta-Kawasaki and Modica-Mortola energies, and more adherent to the Peierls-Nabarro model. Specifically, the asymptotic behaviour of the functionals introduced in (6) and (7) as $\varepsilon \rightarrow 0$ deserves, in our opinion, further investigation.

\section{Heuristic derivation and related models}

In this section we present our model and provide its heuristic derivation from basic semidiscrete models in elasticity. 

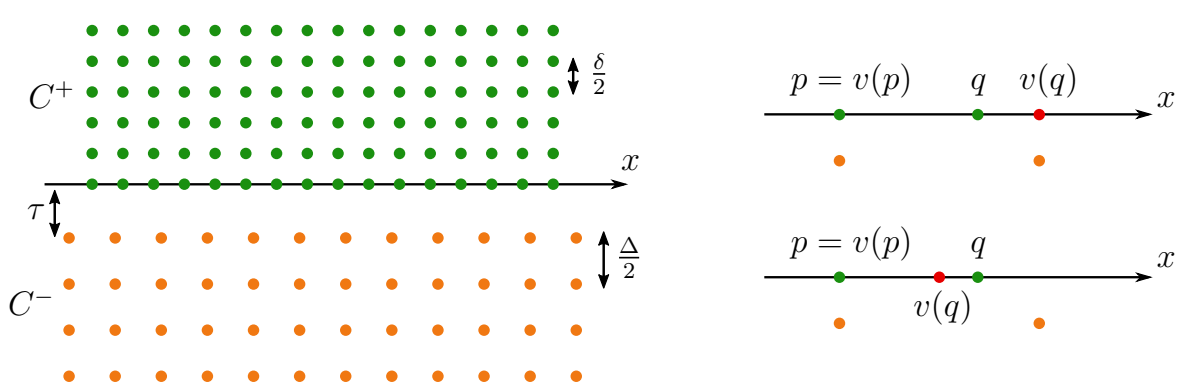

Fig. 1 Left: reference configuration. Top right: purely elastic deformation, with relative displacement $(\Delta-$ $\delta) / 2$. Bottom right: deformation leading to an edge dislocation, with relative displacement $\frac{\Delta}{4}-\frac{\delta}{2}$

\subsection{Semi-coherent interface}

Consider two square lattices $C^{ \pm}$with different lattice spacing occupying the lower and upper half-plane, respectively. More precisely, let $\tau>0$, let $0<\delta<\Delta$, and let

$$
C^{-}:=\frac{\Delta}{2}(\mathbb{Z} \times(-\mathbb{N}))-(0, \tau), \quad C^{+}:=\frac{\delta}{2}(\mathbb{Z} \times \mathbb{N}) .
$$

Here $\frac{\Delta}{2}$ and $\frac{\delta}{2}$ are the lattice spacing of $C^{-}$and $C^{+}$, respectively, and the convenience of the prefactor $\frac{1}{2}$ will be commented later on (Fig. 1). We are assuming higher density for the upper crystal $C^{+}$; nothing would change in our considerations if we assume instead that $C^{+}$ has lower density than $C^{-}$. Clearly, the case of a single crystal corresponds to $\delta=\Delta=\tau$.

The first mathematical simplification in our model consists in assuming that $C^{-}$is rigid, while $C^{+}$has a linear elastic behavior. Our approach consists in focussing on the position of the atoms on $C^{+}$lying on the $x$-axis $\{y=0\}$, which in turn determines the position of all the other atoms in $C^{+}$by elastic energy minimization. More precisely, we assume that each atom $p$ lying on the $x$-axis, can move only along the $x$-axis, and positions itself, after being displaced, either on top of some atom of $C^{-}$, or in the middle of two adjacent atoms in $C^{-}$ (see Fig. 1). The latter case represents an edge dislocation in our model. Moreover, in order to restore the lattice structure where a dislocation is present, we need to somehow complete the dislocation: we assume that two adjacent atoms cannot both be dislocation points; in this way, before and after each dislocation point we have a perfect matching between the two lattices. We also assume that the deformation preserves orientation and that the distance between two adjacent atoms remains strictly larger than 0 and smaller than $\frac{\Delta}{2}$.

It is convenient to describe the deformed configurations of the atoms on the axis $\{y=0\}$ through a displacement function $u: \frac{\delta}{2} \mathbb{Z} \rightarrow \mathbb{R}$. For what has been said so far, we have that if $p$ and $q$ are two adjacent points on the $x$-axis, $p$ to the left of $q$, then $u(q)-u(p)$ can be either $(\Delta-\delta) / 2$ or $\frac{\Delta}{4}-\frac{\delta}{2}$ (see Fig. 1). If we consider the piece-wise affine extension of $u$ on the whole $\mathbb{R}$, this means that

$$
u^{\prime} \in\left\{\lambda:=\frac{\Delta-\delta}{\delta},-\Lambda:=\frac{\Delta}{2 \delta}-1\right\} .
$$

Assuming that the interface between the two crystals is semi-coherent, namely that $\delta \approx \Delta$, yields $0<\lambda \ll 1$, while $\Lambda \approx \frac{1}{2}$. We also notice that the minimal interval where $u^{\prime}$ is constant has length equal to $\frac{\delta}{2}$, and in fact $\delta$ if $u^{\prime}=-\Lambda$. In our model we will partially keep memory of this important fact, enforcing that the region where $u^{\prime}=-\Lambda$ is given by a disjoint union 
of intervals $\left(x_{i}-\frac{\delta}{2}, x_{i}+\frac{\delta}{2}\right)$ of size $\delta$; here the points $x_{i}$ represent edge dislocations, while $\delta$ is proportional to the lattice spacing, as well as to the size of the Burgers vector and of the core region. In order to relax the stored elastic energy towards its ground state, we expect to deal with zero average displacements $u$; this fact, together with assumption $\lambda \ll \Lambda$, yields that the average distance between dislocations is much larger than $\delta$. In this respect, the information that the region where $u^{\prime}=\lambda$ is union of intervals of size $\frac{\delta}{2}$ seems to be less relevant, and will be neglected in our model.

\subsection{The stored elastic energy}

We pass to describe the elastic energy stored in the crystal, in the continuous framework of linearized elasticity. Given a displacement $u$ defined on the $x$-axis, one should consider as admissible any displacement $U: \mathbb{R} \times \mathbb{R}^{+} \rightarrow \mathbb{R}^{2}$ agreeing with $u$ on the $x$-axis. Then, the stored elastic energy density would be a quadratic form $\left\langle\mathbb{C} E_{U}: E_{U}\right\rangle$ where $\mathbb{C}$ denotes the fourth-order elasticity tensor, and $E_{U}$ is the symmetrized gradient of $U$. Instead, here we make the second relevant mathematical simplification of the model (after having assumed $C^{-}$rigid): we replace the canonical elastic energy density with the simpler Dirichlet one, given by $|D U|^{2}$. Formally, the energy stored in the crystal is then

$$
E(U)=\int_{\mathbb{R} \times \mathbb{R}^{+}}|D U|^{2} d z .
$$

This energy is, in principle, unbounded. However let us ignore this fact for a while and formally minimize (5) with respect to all $U$ compatible with $u$. This procedure yields the minimal energy induced by $u$, and in turn by the given configuration of dislocations. The minimizer $U_{\min }$ has only horizontal component, i.e., $U_{\min }=\left(U_{1}, 0\right)$ and, still formally,

$$
E\left(U_{\min }\right)=\int_{\mathbb{R} \times \mathbb{R}^{+}}\left|D U_{1}\right|^{2} d z=c\|u\|_{\dot{H}^{1 / 2}}^{2}=c \int_{\mathbb{R}} \int_{\mathbb{R}} \frac{|u(x)-u(y)|^{2}}{|x-y|^{2}} d x d y,
$$

where $c$ is a suitable pre-factor (see [19]). Clearly, in view of our constraints on $u$, such an energy is always infinite. Therefore, we introduce a length-scale $l>0$, and for $u:(0, l) \rightarrow \mathbb{R}$ define the corresponding elastic energy functionals

$$
E^{l}(u)=\int_{0}^{l} \int_{0}^{l} \frac{|u(x)-u(y)|^{2}}{|x-y|^{2}} d x d y .
$$

The above energy is finite, and diverges with order $l$ as $l \rightarrow+\infty$. Eventually we re-scale $E^{l}$, multiplying it by $\frac{1}{l}$, and consider its limit in the sense of $\Gamma$-convergence as $l \rightarrow+\infty$.

\subsection{Comparison with Peierls-Nabarro models}

In order to establish a comparison between our model and Peierls-Nabarro type models, we need to assume that we are dealing with a single crystal, i.e., $\delta=\Delta$. In such case, we have $\lambda=0$, while $\Lambda=\frac{1}{2}$. Let moreover $u: \mathbb{R} \rightarrow \mathbb{R}$ be the prescribed displacement function at the $x$-axis. Up to an additive constant, we can always assume that $u(0)=0$. Therefore, the function $u$ is made of affine pieces, where $u^{\prime}=-\Lambda$, and flat regions, where $u$ takes values in $\delta \Lambda \mathbb{Z}=\frac{\delta}{2} \mathbb{Z}$. Again, the regions where $u^{\prime}=-\Lambda$ can be identified with the dislocation cores, and $u^{\prime} \equiv-\Lambda$ can be understood as a plastic strain. As we will see in Theorem 2.6, dislocations arise as energy minimizers, and are induced only by the lattice misfit: In this 
respect, the corresponding strain $u^{\prime} \equiv-\Lambda$ is usually referred to as eigenstrain, as it is produced without external forces; we refer the interested reader to [4].

Here the model is quite rigid, prescribing exactly the values of the strains in the dislocation cores, and a perfect lattice matching $u(x) \in \frac{\delta}{2} \mathbb{Z}$ outside the cores. In this respect, the configurations considered in this model are more rigid with respect to the classical PeierlsNabarro model [26], but consistent with their analysis showing that the size of a dislocation is of the order of few lattice spacings. Relaxing these conditions gives back the celebrated Peierls-Nabarro model, where the condition $u(x) \in \frac{\delta}{2} \mathbb{Z}$ is enforced by a potential with wells exactly at $\frac{\delta}{2} \mathbb{Z}$.

In this respect, our model can be regarded as a Peierls-Nabarro model for heterogeneous crystals, whithin the more rigid formalism of eigenstrains.

On the other hand, one could also consider models which are more closely related to the Peierls-Nabarro formalism. For instance, given $\varepsilon>0$ and noticing that $(\lambda+\Lambda) \delta=\frac{\Delta}{2}$, one could consider the functional

$$
E_{\varepsilon}(u):=\varepsilon\|u\|_{\dot{H}^{\frac{1}{2}}}^{2}+\frac{1}{\varepsilon} \int_{\mathbb{R}} \operatorname{dist}^{2}\left(u(x)-\lambda x, \frac{\Delta}{2} \mathbb{Z}\right) d x .
$$

Setting $f(x):=u(x)-\lambda x$, the energy can be written as

$$
E_{\varepsilon}(f):=\varepsilon\|f(x)+\lambda x\|_{\dot{H}^{\frac{1}{2}}}^{2}+\frac{1}{\varepsilon} \int_{\mathbb{R}} \operatorname{dist}^{2}\left(f(x), \frac{\Delta}{2} \mathbb{Z}\right) d x .
$$

Here $\varepsilon$ fixes the length-scale of the transitions, so that for $\varepsilon \approx \delta$ we expect that the minimizers of the functionals $E_{\varepsilon}$ behave similarly to the ones of our proposed model. If on the one hand these energies $E_{\varepsilon}$ seem the natural counterpart of Peierls-Nabarro functionals for heterogeneous crystals, our proposed model seems, at a first glance, more feasible and easier to be analyzed.

\subsection{Comparison with the Ohta-Kawasaki model}

The Ohta-Kawasaki variational model, in its basic form, consists in minimizing energy functionals acting on scalar functions $u$ and which are given by the sum of the $H^{-1}$ norm of $u$ and a two wells potential forcing $u$ to take values in $\{ \pm 1\}$. Such energy is completed with some extra terms, which prescribe the scale at which the oscillations of $u$ occur.

In fact, it is well known that minimizers exhibit periodic oscillations; the first rigorous proof of this behavior has been carried out in dimension one in [20]. There, it is introduced (up to suitable equivalent reformulation) the following energy

$$
\|v\|_{H^{-1}}^{2}+\varepsilon^{2}\left\|v^{\prime}\right\|_{2}^{2}+\left\|v^{2}-1\right\|_{2}^{2}
$$

and it is proven that, for $\varepsilon$ small enough, minimizers are periodic.

Our model is somehow based on minimization of the analogous energy functional where the $H^{-1}$ norm replaced by the $H^{-\frac{1}{2}}$ one. In fact, consider the energy

$$
\|v\|_{H^{-\frac{1}{2}}}^{2}+\varepsilon^{2}\left\|v^{\prime}\right\|_{2}^{2}+\left\|v^{2}-1\right\|_{2}^{2} \text {. }
$$

Setting $u^{\prime}:=v$ in the above expression leads to the minimization of following functional

$$
\|u\|_{H^{\frac{1}{2}}}^{2}+\varepsilon^{2}\left\|u^{\prime \prime}\right\|_{2}^{2}+\left\|\left(u^{\prime}\right)^{2}-1\right\|_{2}^{2} .
$$

This energy is strictly related to our model for $\lambda=\Lambda=1$. In fact, the third term forces $u^{\prime}$ to take values in $\{ \pm 1\}$, while the second fixes the scale of transitions between such phases, 
and therefore, together with the first term, determines their number. In our proposed model, the length and number of transitions is enforced replacing the above penalizations with a constraint on the minimal length of the phases. In this respect, it seems interesting to consider the following relaxed version of our energy, more closely related with the Ohta-Kawasaki formalism

$$
\|u\|_{H^{\frac{1}{2}}}^{2}+\varepsilon^{2}\left\|u^{\prime \prime}\right\|_{2}^{2}+\left\|\operatorname{dist}\left(u^{\prime},\{\lambda,-\Lambda\}\right)\right\|_{2}^{2},
$$

or, equivalently

$$
\|v\|_{H^{-\frac{1}{2}}}^{2}+\varepsilon^{2}\left\|v^{\prime}\right\|_{2}^{2}+\|\operatorname{dist}(v,\{\lambda,-\Lambda\})\|_{2}^{2} .
$$

\section{The mathematical model}

\subsection{Admissible configurations and their energy}

Let $\lambda, \Lambda>0$ with $\Lambda \geq \lambda$; let moreover $\delta>0$ be fixed, and $l \geq 0$. We introduce the family of admissible dislocations as

$$
\begin{aligned}
\mathcal{A D}_{l}:= & \left\{\left\{x_{1}, \ldots, x_{N}\right\} \subset\left(-\frac{\delta}{2}, l+\frac{\delta}{2}\right) \text { with } N \in \mathbb{N}, x_{1}<x_{2} \ldots<x_{N},\right. \\
& \text { and with } \left.\left(x_{i}-\frac{\delta}{2}, x_{i}+\frac{\delta}{2}\right) \text { mutually disjoint }\right\} .
\end{aligned}
$$

Notice that dislocations can fall outside of $(0, l)$, and that the dislocation set can also be empty, namely $\emptyset \in \mathcal{A D}_{l}$. Given $X \in \mathcal{A D}_{l}$, we consider the corresponding displacement at interface $u_{X} \in W^{1, \infty}(0, l)$ determined, up to an additive constant, by

$$
u_{X}^{\prime}=\lambda-(\Lambda+\lambda) \chi_{(0, l)} \sum_{i=1}^{N} \chi_{\left(x_{i}-\frac{\delta}{2}, x_{i}+\frac{\delta}{2}\right)} .
$$

The class of admissible displacements is given by

$$
\mathcal{U}_{l}:=\left\{u_{X}, X \in \mathcal{A D}_{l}\right\} .
$$

The energy $E^{l}(u)$ associated to any $u \in \mathcal{U}_{l}$ is nothing but the square of its $H^{\frac{1}{2}}$-seminorm:

$$
E^{l}(u):=\|u\|_{\dot{H}^{1 / 2}}^{2}=\int_{0}^{l} \int_{0}^{l} \frac{|u(x)-u(y)|^{2}}{|x-y|^{2}} d x d y .
$$

Clearly, the energy diverges as $l \rightarrow+\infty$, therefore we need a suitable rescaling. We will now provide an estimate for the energy of a function whose oscillation is controlled by a constant.

Lemma 2.1 Given $M>0$ there exists $C>0$ such that, for every $l>1$ and for all $u \in \mathcal{U}_{l}$ satisfying

$$
\max \{|u(x)-u(y)|: x, y \in(0, l)\} \leq M,
$$

we have

$$
E^{l}(u) \leq C l
$$


Proof Let us set $A:=\left\{(x, y) \in[0, l]^{2}:|x-y| \leq 1\right\}$ and $B:=A^{c} \cap[0, l]^{2}$. Thus

$$
E^{l}(u)=\iint_{A} \frac{|u(x)-u(y)|^{2}}{|x-y|^{2}} d x d y+\iint_{B} \frac{|u(x)-u(y)|^{2}}{|x-y|^{2}} d x d y
$$

and since $u$ is $\Lambda$-Lipschitz we get

$$
\iint_{A} \frac{|u(x)-u(y)|^{2}}{|x-y|^{2}} d x d y \leq \Lambda^{2}|A| \leq 2 \Lambda^{2} l .
$$

Let us estimate the second integral in (12); using (11) we have

$$
\begin{aligned}
\iint_{B} \frac{|u(x)-u(y)|^{2}}{|x-y|^{2}} d x d y & \leq \iint_{B} \frac{M^{2}}{|x-y|^{2}} d x d y=2 M^{2} \int_{1}^{l} \int_{0}^{x-1} \frac{1}{|x-y|^{2}} d y d x \\
& =2 M^{2}(l-1-\log l) \leq 2 M^{2} l
\end{aligned}
$$

for each $l>1$. The thesis is achieved with $C:=2 M^{2}+2 \Lambda^{2}$.

Remark 2.2 (Energy for evenly spaced dislocations) We want to show that the energy $E^{l}$ for a sequence $u_{l} \in \mathcal{U}_{l}$ inducing a periodic network of dislocations grows at most linearly in $l$. Set $\gamma:=\frac{\lambda+\Lambda}{\lambda} \delta, N_{l}:=\left\lfloor\frac{l}{\gamma}\right\rfloor$ and $r(\gamma, l):=l-N_{l} \gamma$ so that $0<r(\gamma, l)<\gamma$. The integer $N_{l}$ represents the number of dislocations which will be present in $(0, l)$. Define intervals

$$
I_{i}:=((i-1) \gamma, i \gamma-\delta), \quad J_{i}:=(i \gamma-\delta, i \gamma), \quad R:=\left(N_{l} \gamma, l\right)
$$

for $i=1, \ldots, N_{l}$, hence obtaining a partition of $(0, l)$. Define $u_{l}$ as the map such that $u_{l}(0)=u_{l}\left(N_{l} \gamma\right)=0, u_{l}^{\prime}=\lambda$ on $I_{i}$ and $R, u_{l}^{\prime}=-\Lambda$ on $J_{i}$. This is possible since, thanks to the choice of $\gamma$, one can check that $\int_{0}^{N_{l} \gamma} u_{l}^{\prime}(x) d x=0$. In this way $u_{l} \in \mathcal{U}_{l}$ and the dislocations are evenly spaced.

Notice that the maximum oscillation of $u_{l}$ in $\left(0, N_{l} \gamma\right)$ is exactly $\Lambda \delta$; namely

$$
\max \left\{|u(x)-u(y)|: x, y \in\left(0, N_{l} \gamma\right)\right\}=\Lambda \delta .
$$

In the interval $R$ the oscillation is given by $\lambda|R|$, and

$$
\lambda|R|=\lambda r(\gamma, l)<\lambda \gamma=\Lambda \delta+\lambda \delta<2 \Lambda \delta,
$$

thanks to the assumption $\lambda \leq \Lambda$. From (14) we then deduce

$$
\max \{|u(x)-u(y)|: x, y \in(0, l)\}<2 \Lambda \delta .
$$

Therefore from Lemma 2.1 we conclude that $E^{l}\left(u_{l}\right)$ scales like $l$.

In view of the above remark, we will rescale $E^{l}$, dividing it by $l$. Exploiting the change of variables $x^{\prime}=l x, y^{\prime}=l y$ the energy reads

$$
E^{l}(u)=\int_{0}^{1} \int_{0}^{1} \frac{|u(l x)-u(l y)|^{2}}{|x-y|^{2}} d x d y .
$$

We introduce the class of admissible rescaled displacements $\mathcal{W}_{l} \subset W^{1, \infty}(0,1)$,

$$
\mathcal{W}_{l}:=\left\{w_{u}(z):=\frac{u(l z)}{\sqrt{l}}, u \in \mathcal{U}_{l}\right\} .
$$

Setting

$$
F^{l}(w):=\int_{0}^{1} \int_{0}^{1} \frac{|w(x)-w(y)|^{2}}{|x-y|^{2}} d x d y \quad \text { for all } w \in \mathcal{W}_{l},
$$


the energy can be written as

$$
E^{l}(u)=l F^{l}\left(w_{u}\right) .
$$

Notice that, given $w \in \mathcal{W}_{l}$, there exists $X=X_{w} \in \mathcal{A D}_{l}$ and $u_{X} \in \mathcal{U}_{l}$ satisfying (9) such that

$$
w=w_{u_{X}} .
$$

\subsection{Asymptotic behavior of the energy functionals}

For each $l>0$ define

$$
c_{l}:=\min _{u \in \mathcal{U}_{l}} \frac{1}{l} \int_{0}^{l} \int_{0}^{l} \frac{|u(x)-u(y)|^{2}}{|x-y|^{2}} d x d y .
$$

Theorem 2.3 There exists $0<c_{\infty}<+\infty$ such that

$$
\lim _{l \rightarrow+\infty} c_{l}=c_{\infty} .
$$

Proof First notice that there exists $C>0$ such that

$$
\sup _{l>1} c_{l} \leq C \text {. }
$$

This follows by choosing the maps $u_{l}$ defined in Remark 2.2 as competitors for $c_{l}$.

Now, for each $l$, let $u_{l} \in \mathcal{U}_{l}$ be a minimizer for $c_{l}$ defined in (19) (whose existence follows by the standard direct method). Fix $h>0$. For each $l>h$ we define intervals $I_{i}:=$ $(h(i-1), h i)$ for $i=1, \ldots, N$, where $N:=\lfloor l / h\rfloor$. Define the remainder $r(h, l):=l-h N$ and notice that $0 \leq r(h, l)<h$. We can choose an interval $I_{j}$ such that

$$
\int_{I_{j}} \int_{I_{j}} \frac{\left|u_{l}(x)-u_{l}(y)\right|^{2}}{|x-y|^{2}} d x d y \leq \frac{1}{N} \sum_{i=1}^{N} \int_{I_{i}} \int_{I_{i}} \frac{\left|u_{l}(x)-u_{l}(y)\right|^{2}}{|x-y|^{2}} d x d y .
$$

Since $u_{l}$ is a competitor for $c_{h}$ on $I_{j}$, we can estimate

$$
\begin{aligned}
c_{h} & \leq \frac{1}{h} \int_{I_{j}} \int_{I_{j}} \frac{\left|u_{l}(x)-u_{l}(y)\right|^{2}}{|x-y|^{2}} d x d y \leq \frac{1}{h N} \sum_{i=1}^{N} \int_{I_{i}} \int_{I_{i}} \frac{\left|u_{l}(x)-u_{l}(y)\right|^{2}}{|x-y|^{2}} d x d y \\
& \leq \frac{1}{h N} \int_{0}^{l} \int_{0}^{l} \frac{\left|u_{l}(x)-u_{l}(y)\right|^{2}}{|x-y|^{2}} d x d y=\frac{l}{h N} c_{l}=\frac{l}{l-r(h, l)} c_{l}
\end{aligned}
$$

Set $\underline{c}:=\liminf _{l \rightarrow+\infty} c_{l}$ and $\bar{c}:=\limsup _{l \rightarrow+\infty} c_{l}$, and recall that $\bar{c}<+\infty$.

Let $\left\{l_{n}\right\},\left\{L_{n}\right\}$ be such that $c_{l_{n}} \geq \bar{c}-\frac{1}{n}, c_{L_{n}} \leq \underline{c}+\frac{1}{n}$ for every $n \in \mathbb{N}$ and $\frac{L_{n}}{l_{n}} \rightarrow+\infty$ as $n \rightarrow+\infty$. By (21) with $h$ replaced by $l_{n}$ and $l$ replaced by $L_{n}$, we have

$$
\bar{c}-\frac{1}{n} \leq c_{l_{n}} \leq \frac{L_{n}}{L_{n}-r\left(l_{n}, L_{n}\right)} c_{L_{n}} \leq \frac{L_{n}}{L_{n}-r\left(l_{n}, L_{n}\right)}\left(\underline{c}+\frac{1}{n}\right) .
$$

Since $r\left(l_{n}, L_{n}\right)$ is bounded by $l_{n}$ and recalling that $\frac{L_{n}}{l_{n}} \rightarrow+\infty$, by taking the limit as $n \rightarrow+\infty$ in the above inequalities we obtain $\bar{c} \leq \underline{c}$, and we clearly deduce that equality holds and denote by $c_{\infty}:=\bar{c}=\underline{c}$ such a quantity.

Finally, we show that $c_{\infty}$ is positive: By the condition on the derivatives of maps belonging to $\mathcal{U}_{1}$, it is immediate to see that $c_{1}>0$. By setting $h=1$ in (21) and letting $l \rightarrow+\infty$ we then infer that $c_{\infty}>0$. 
We can now define the candidate $\Gamma$-limit for $F^{l}$ as

$$
F^{\infty}(w):= \begin{cases}\int_{0}^{1} \int_{0}^{1} \frac{|w(x)-w(y)|^{2}}{|x-y|^{2}}+c_{\infty} & \text { if } w \in H^{\frac{1}{2}}(0,1) \\ +\infty & \text { otherwise }\end{cases}
$$

where the constant $c_{\infty}$ is defined in Theorem 2.3.

As a consequence of Theorem 2.3 we also obtain the following corollary:

Corollary 2.4 For any $l>0$ let $x=x_{l} \in[0, l]$ be an arbitrary point. Let $u_{l} \in \mathcal{U}_{l}$ be a minimizer for $c_{l}$. Then

$$
\lim _{l \rightarrow+\infty} \frac{1}{l} \int_{0}^{x_{l}} \int_{x_{l}}^{l} \frac{\left|u_{l}(x)-u_{l}(y)\right|^{2}}{|x-y|^{2}} d x d y=0 .
$$

Proof By Theorem 2.3 the function $s \mapsto R(s):=c_{s}-c_{\infty}$ is bounded and such that $R(s) \rightarrow 0$ as $s \rightarrow+\infty$. Therefore, for every sequence $\left\{x_{l}\right\}_{l}$ we easily infer

$$
\frac{x_{l}}{l} R\left(x_{l}\right) \rightarrow 0, \quad \frac{l-x_{l}}{l} R\left(l-x_{l}\right) \rightarrow 0, \quad \text { as } l \rightarrow+\infty .
$$

Having said that, we write

$$
\begin{aligned}
c_{l}= & \frac{1}{l} \int_{0}^{x_{l}} \int_{0}^{x_{l}} \frac{\left|u_{l}(x)-u_{l}(y)\right|^{2}}{|x-y|^{2}} d x d y+\frac{1}{l} \int_{x_{l}}^{l} \int_{x_{l}}^{l} \frac{\left|u_{l}(x)-u_{l}(y)\right|^{2}}{|x-y|^{2}} d x d y \\
& +\frac{2}{l} \int_{0}^{x_{l}} \int_{x_{l}}^{l} \frac{\left|u_{l}(x)-u_{l}(y)\right|^{2}}{|x-y|^{2}} d x d y \\
\geq & \frac{x_{l}}{l} c_{x_{l}}+\frac{l-x_{l}}{l} c_{l-x_{l}}+\frac{2}{l} \int_{0}^{x_{l}} \int_{x_{l}}^{l} \frac{\left|u_{l}(x)-u_{l}(y)\right|^{2}}{|x-y|^{2}} d x d y \\
= & c_{\infty}+\frac{x_{l}}{l} R\left(x_{l}\right)+\frac{l-x_{l}}{l} R\left(l-x_{l}\right)+\frac{2}{l} \int_{0}^{x_{l}} \int_{x_{l}}^{l} \frac{\left|u_{l}(x)-u_{l}(y)\right|^{2}}{|x-y|^{2}} d x d y,
\end{aligned}
$$

and taking the limsup as $l \rightarrow+\infty$ entails

$$
c_{\infty} \geq c_{\infty}+\limsup _{l \rightarrow+\infty} \frac{2}{l} \int_{0}^{x_{l}} \int_{x_{l}}^{l} \frac{\left|u_{l}(x)-u_{l}(y)\right|^{2}}{|x-y|^{2}} d x d y \geq c_{\infty},
$$

from which the thesis follows.

Proposition 2.5 Let $u_{l} \in \mathcal{U}_{l}$ be a minimizer of problem (19) for each $l>0$, and let $w_{l}:=w_{u_{l}}$ be defined as in (16) and $a_{l}:=\int_{0}^{1} w_{l} d x$. Then

$$
\left(w_{l}-a_{l}\right) \rightarrow 0 \text { weakly in } H^{\frac{1}{2}}(0,1) \text { as } l \rightarrow+\infty \text {. }
$$

Proof Set $\tilde{w}_{l}:=w_{l}-a_{l}$. By Theorem 2.3 one can easily check that $\tilde{w}_{l}$ has bounded $\dot{H}^{\frac{1}{2}}(0,1)$ seminorm; therefore, by Poincaré-Wirtinger inequality, up to a subsequence, $\tilde{w}_{l} \rightarrow \tilde{w}$ in $H^{\frac{1}{2}}(0,1)$. Moreover, by the change of variables $x^{\prime}=l x, y^{\prime}=l y$ and by Corollary 2.4 applied to $x_{l}=l / 2$ we infer

$$
\int_{0}^{\frac{1}{2}} \int_{\frac{1}{2}}^{1} \frac{\left|\tilde{w}_{l}(x)-\tilde{w}_{l}(y)\right|^{2}}{|x-y|^{2}} d x d y=\frac{1}{l} \int_{0}^{\frac{l}{2}} \int_{\frac{l}{2}}^{l} \frac{\left|u_{l}(x)-u_{l}(y)\right|^{2}}{|x-y|^{2}} d x d y \rightarrow 0,
$$


as $l \rightarrow+\infty$. By lower semi-continuity we deduce that

$$
\int_{0}^{\frac{1}{2}} \int_{\frac{1}{2}}^{1} \frac{|\tilde{w}(x)-\tilde{w}(y)|^{2}}{|x-y|^{2}} d x d y=0,
$$

which clearly implies that $\tilde{w}$ is constant, and in fact, since it has zero average, $\tilde{w} \equiv 0$. We conclude that the whole sequence $w_{l}-a_{l}$ weakly converges to 0 in $H^{\frac{1}{2}}(0,1)$.

Finally, the fact that minimizers weakly converge to zero leads to the fact that optimal dislocation configurations tend to be uniformly distributed in the limit as $l \rightarrow+\infty$.

Theorem 2.6 Let $w_{l}$ be a family of minimizers of $F^{l}$, and let $X_{l}=\left\{x_{1}, \ldots, \ldots x_{N_{l}}\right\}$ be the corresponding family of configurations of dislocations defined as in (18). Then, setting $\mu_{l}:=\frac{1}{l} \sum_{i=1}^{N_{l}} \delta \frac{x_{i}}{l}$ we have that $\mu_{l} \stackrel{*}{\rightarrow} \frac{\Lambda}{\delta(\Lambda+\lambda)}$ as $l \rightarrow+\infty$.

Proof Let us fix $0<p<q<1$; by Proposition 2.5 we have that, up to an additive constant, $w_{l} \rightarrow 0$ in $L^{1}$. Therefore, there exist $p_{l} \rightarrow p, q_{l} \rightarrow q$ such that $r_{l}:=w_{l}\left(q_{l}\right)-w_{l}\left(p_{l}\right) \rightarrow 0$ as $l \rightarrow+\infty$. Then, setting $M_{l}:=\sharp \frac{X_{l}}{l} \cap\left(p_{l}, q_{l}\right)$ we have

$$
r_{l}=w_{l}\left(q_{l}\right)-w_{l}\left(p_{l}\right)=\int_{p_{l}}^{q_{l}} w_{l}^{\prime} d x=-\frac{M_{l}}{\sqrt{l}}(\Lambda+\lambda) \delta+\lambda \sqrt{l}\left(q_{l}-p_{l}\right)+\tilde{r}_{l},
$$

where $\tilde{r}_{l} \rightarrow 0$ as $l \rightarrow+\infty$. We deduce that

$$
\frac{M_{l}}{l}=\frac{\lambda}{\delta(\Lambda+\lambda)}(q-p)+\hat{r}_{l},
$$

where $\hat{r}_{l} \rightarrow 0$ as $l \rightarrow+\infty$. By the arbitrariness of $p$ and $q$ in $(0,1)$, we conclude the thesis.

\section{3 Г-convergence}

Theorem 3.1 [ $\Gamma$-convergence] As $l \rightarrow+\infty$, the functionals $F^{l}$ defined in (17) $\Gamma$-converge with respect to the weak topology of $H^{\frac{1}{2}}(0,1)$ to the functional $F^{\infty}$, defined in (22).

\subsection{Proof of $\Gamma$-liminf inequality}

Let $w_{l} \in \mathcal{W}_{l}$ be such that $w_{l} \rightarrow w$ weakly in $H^{\frac{1}{2}}$. Let $l \mapsto M_{l} \in(0, l) \cap \mathbb{N}$ be such that $M_{l} \rightarrow+\infty$ as $l \rightarrow+\infty$ and

$$
\lim _{l \rightarrow+\infty} \frac{M_{l}}{l}=0
$$

Let $x_{i}:=\frac{i}{M_{l}}$ for $i=0, \ldots, M_{l}$ and set $I_{i}:=\left(x_{i-1}, x_{i}\right)$ for $i=1, \ldots, M_{l}$. In order to show the $\Gamma$-liminf inequality, we decompose the energy as

$$
\begin{aligned}
\left\|w_{l}\right\|_{\dot{H}^{\frac{1}{2}}}^{2} & =\sum_{i \neq j}^{M_{l}} \int_{I_{i}} \int_{I_{j}} \frac{\left|w_{l}(x)-w_{l}(y)\right|^{2}}{|x-y|^{2}} d x d y+\sum_{i=1}^{M_{l}} \int_{I_{i}} \int_{I_{i}} \frac{\left|w_{l}(x)-w_{l}(y)\right|^{2}}{|x-y|^{2}} d x d y \\
& =: J_{l}^{1}+J_{l}^{2} .
\end{aligned}
$$


Let us first estimate $J_{l}^{2}$. For $x \in\left(0, l / M_{l}\right)$ and each $i=1, \ldots, M_{l}$, define the rescaled function

$$
u_{l}^{i}(x):=\sqrt{l} w_{l}\left(\frac{x}{l}+x_{i-1}\right) .
$$

By its very definition $u_{l}^{i} \in \mathcal{U}_{\frac{l}{M_{l}}}$, and whence it is a competitor for $c_{\frac{l}{M_{l}}}$, as defined in (19). Therefore, by introducing the new variables $x=x^{\prime} / l+x_{i-1}, y=y^{\prime} / l+x_{i-1}$ and recalling that $x_{i}=x_{i-1}+1 / M_{l}$, we infer

$$
\begin{aligned}
& \int_{I_{i}} \int_{I_{i}} \frac{\left|w_{l}(x)-w_{l}(y)\right|^{2}}{|x-y|^{2}} d x d y \\
& =\int_{0}^{l / M_{l}} \int_{0}^{l / M_{l}} \frac{\left|w_{l}\left(\frac{x^{\prime}}{l}+x_{i-1}\right)-w_{l}\left(\frac{y^{\prime}}{l}+x_{i-1}\right)\right|^{2}}{\left|x^{\prime}-y^{\prime}\right|^{2}} d x^{\prime} d y^{\prime} \\
& =\frac{1}{l} \int_{0}^{l / M_{l}} \int_{0}^{l / M_{l}} \frac{\left|u_{l}^{i}\left(x^{\prime}\right)-u_{l}^{i}\left(y^{\prime}\right)\right|^{2}}{\left|x^{\prime}-y^{\prime}\right|^{2}} d x^{\prime} d y^{\prime} \geq \frac{1}{M_{l}} c_{\frac{l}{M_{l}}}
\end{aligned}
$$

Hence

$$
J_{l}^{2} \geq c_{\frac{l}{M_{l}}} .
$$

By assumption (25) we have $l / M_{l} \rightarrow+\infty$ as $l \rightarrow+\infty$, therefore we can apply Theorem 2.3 and conclude that

$$
\liminf _{l \rightarrow+\infty} J_{l}^{2} \geq \lim _{l \rightarrow+\infty} c_{\frac{l}{M_{l}}}=c_{\infty} .
$$

From the above inequality we get

$$
\liminf _{l \rightarrow+\infty}\left\|w_{l}\right\|_{\dot{H}^{\frac{1}{2}}}^{2}=\liminf _{l \rightarrow+\infty}\left(J_{l}^{1}+J_{l}^{2}\right) \geq \liminf _{l \rightarrow+\infty} J_{l}^{1}+c_{\infty} .
$$

We are left to estimate $J_{l}^{1}$, and we have to prove that

$$
\liminf _{l \rightarrow+\infty} J_{l}^{1} \geq\|w\|_{\dot{H}^{\frac{1}{2}}}^{2} .
$$

Let $Q:=[0,1] \times[0,1], D_{l}:=\cup_{i=1}^{M_{l}} I_{i} \times I_{i}$ and $Q_{l}:=Q \backslash D_{l}$. For a.e. $(x, y) \in Q$ define

$$
g(x, y):=\frac{|w(x)-w(y)|}{|x-y|}, \quad g_{l}(x, y):=\frac{\left|w_{l}(x)-w_{l}(y)\right|}{|x-y|}, \quad \tilde{g}_{l}:=\chi_{Q_{l}} g_{l} .
$$

Now notice that, as $l \rightarrow+\infty$,

$$
g_{l} \rightarrow g \quad \text { weakly in } \quad L^{2}(Q) .
$$

Indeed, since we are assuming that $w_{l} \rightarrow w$ weakly in $H^{\frac{1}{2}}(0,1)$, by compact Sobolev embedding, one has that $\left(w_{l}-\int_{0}^{1} w_{l} d x\right) \rightarrow\left(w-\int_{0}^{1} w d x\right)$ strongly in $L^{2}(0,1)$ (by a Poincaré-Wirtinger type inequality we can control the full norm of $\left(w_{l}-\int_{0}^{1} w_{l} d x\right)$ in $\left.H^{\frac{1}{2}}\right)$. Therefore, up to subsequences, we also have $\left(w_{l}-\int_{0}^{1} w_{l} d x\right) \rightarrow\left(w-\int_{0}^{1} w d x\right)$ a.e. in $(0,1)$. By definition we then have $g_{l} \rightarrow g$ a.e. in $Q$. Since $\left\|g_{l}\right\|_{L^{2}(Q)}=\left\|w_{l}\right\|_{\dot{H}^{\frac{1}{2}}}$ is uniformly bounded, we also have (along the subsequence) $g_{l} \rightarrow g$ weakly in $L^{2}(Q)$. Since the limit does not depend on the subsequence, we conclude (28). Observe that $\chi_{Q_{l}} \rightarrow 1$ strongly in $L^{p}(Q)$ for every $1 \leq p<\infty$, since $\left|D_{l}\right|=1 / M_{l} \rightarrow 0$ as $l \rightarrow+\infty$. Therefore from (28) we conclude that $\tilde{g}_{l} \rightarrow g$ weakly in $L^{2}(Q)$, and so (27) follows by lower semicontinuity, upon noticing that $\left\|\tilde{g}_{l}\right\|_{L^{2}(Q)}^{2}=J_{l}^{1}$. From (26), (27) we conclude the $\Gamma$-liminf inequality. 


\subsection{Proof of the $\Gamma$-limsup inequality}

In order to prove the $\Gamma$-limsup inequality, we need the following Lemma.

Lemma 3.2 Let $u_{l}$ be a minimizer for (19). Let moreover $A_{l} \subset[0, l]$ be an open set such that $\left|A_{l}\right| / l \rightarrow 0$ as $l \rightarrow+\infty$, and $A_{l}$ is union of intervals whose length is larger than some constant $C>0$ independent of $l$. Then

$$
\lim _{l \rightarrow+\infty} \frac{1}{l} \int_{A_{l}} \int_{0}^{l} \frac{\left|u_{l}(x)-u_{l}(y)\right|^{2}}{|x-y|^{2}} d x d y=0 .
$$

Proof Let $l_{n} \rightarrow+\infty$ be such that

$$
\begin{aligned}
\lim _{n \rightarrow+\infty} \frac{1}{l_{n}} \int_{A_{l n}} \int_{0}^{l_{n}} \frac{\left|u_{l_{n}}(x)-u_{l_{n}}(y)\right|^{2}}{|x-y|^{2}} d x d y & =\limsup _{l \rightarrow+\infty} \frac{1}{l} \int_{A_{l}} \int_{0}^{l} \frac{\left|u_{l}(x)-u_{l}(y)\right|^{2}}{|x-y|^{2}} d x d y \\
& =: E .
\end{aligned}
$$

By the optimality of $u_{l}$ we have

$$
\lim _{n \rightarrow+\infty} \frac{1}{l_{n}} \int_{A_{l_{n}}^{c}} \int_{0}^{l_{n}} \frac{\left|u_{l_{n}}(x)-u_{l_{n}}(y)\right|^{2}}{|x-y|^{2}} d x d y=c_{\infty}-E,
$$

where we denote by $A_{l_{n}}^{c}$ the complement of $A_{l_{n}}$ in $[0, l]$. Fix $N \in \mathbb{N}$, and let $\left\{B_{i, n}^{N}\right\}_{i}$ be the connected components of $A_{l_{n}}^{c}$ whose length is at least $N$. By the assumption $\left|A_{l}\right| / l \rightarrow 0$ as $l \rightarrow+\infty$, we infer

$$
\lim _{n \rightarrow+\infty} \frac{\sum_{i}\left|B_{i, n}^{N}\right|}{l_{n}}=1
$$

Hence by (29), there exists at least one element of $\left\{B_{i, n}^{N}\right\}_{i}$, which we name $\left(x_{n, N}, y_{n, N}\right)$, such that

$$
\limsup _{n \rightarrow+\infty} \frac{1}{y_{n, N}-x_{n, N}} \int_{x_{n, N}}^{y_{n, N}} \int_{x_{n, N}}^{y_{n, N}} \frac{\left|u_{l_{n}}(x)-u_{l_{n}}(y)\right|^{2}}{|x-y|^{2}} d x d y \leq c_{\infty}-E .
$$

By a diagonal argument, there exists a subsequence $\tilde{l}_{N}=l_{n_{N}}$ and intervals $\left(x_{N}, y_{N}\right)$ such that

$$
\lim _{N \rightarrow+\infty} \frac{1}{y_{N}-x_{N}} \int_{x_{N}}^{y_{N}} \int_{x_{N}}^{y_{N}} \frac{\left|u_{\tilde{l}_{N}}(x)-u_{\tilde{l}_{N}}(y)\right|^{2}}{|x-y|^{2}} d x d y \leq c_{\infty}-E .
$$

Setting $\tilde{u}_{N}(x):=u_{\tilde{l}_{N}}\left(x-x_{N}\right)$ we have

$$
\lim _{N \rightarrow+\infty} \frac{1}{y_{N}-x_{N}} \int_{0}^{y_{N}-x_{N}} \int_{0}^{y_{N}-x_{N}} \frac{\left|\tilde{u}_{N}(x)-\tilde{u}_{N}(y)\right|^{2}}{|x-y|^{2}} d x d y \leq c_{\infty}-E .
$$

Since $\tilde{u}_{N} \in \mathcal{U}_{y_{N}-x_{N}}$ and recalling that $y_{N}-x_{N} \rightarrow+\infty$, by Theorem 2.3 we conclude that $E=0$, ending the proof.

Now, let $w \in H^{\frac{1}{2}}(0,1)$. We want to construct a recovery sequence $g_{l} \in \mathcal{W}_{l}$ such that

$$
\begin{aligned}
& g_{l} \rightarrow w \quad \text { weakly in } H^{\frac{1}{2}}(0,1) \text { as } l \rightarrow+\infty, \\
& \limsup _{l \rightarrow+\infty} F^{l}\left(g_{l}\right)=F^{\infty}(w),
\end{aligned}
$$


where $F^{l}$ is defined in (17) and $F^{\infty}$ in (22).

By standard density arguments in $\Gamma$-convergence we may assume that $w$ is piece-wise affine. Specifically, without loss of generality we assume that $w \in C^{0}(0,1)$ is of the form $w=\sum_{i=1}^{m}\left(c_{i}+\alpha_{i} x\right) \chi_{I_{i}}$ where $c_{i} \in \mathbb{R}, \alpha_{i} \in \mathbb{R} \backslash\{0\}$, and $\left\{I_{i}\right\}$ is a partition of $(0,1)$.

Let $\left\{w_{l}\right\} \subset W^{1, \infty}(0,1)$ be a family of minimizers provided by Proposition 2.5 and with average equal to zero, and let $X_{w_{l}}$ be a set of dislocations associated to $w_{l}$, defined as in (18). Now we want to plug extra dislocations $N_{w_{l}}$, inducing the macroscopic strain $w^{\prime}$. In principle, we need periodically distributed dislocations whose density depends on $\alpha_{i}$. However, some care is needed to ensure that the new dislocations are not plugged on top of the dislocations already present, namely, they should be introduced in such a way that $X_{w_{l}} \cup N_{w_{l}}$ gives back an admissible configuration of dislocations. To this purpose, it is easy to see that there exists a finite family of points $N_{w_{l}}=\left\{x_{1}, \ldots x_{N_{l}}\right\} \subset(0,1)$ with $x_{i}<x_{i+1}$ for all $i$ with the following properties:

i) $|x-z| \geq \frac{\delta}{l}$ for all $x \in N_{w_{l}}, z \in \frac{1}{l} X_{w_{l}}$;

ii) The distance between any pair of consecutive points is prescribed up to errors of order $\frac{\delta}{l}$ as follows:

$$
\mid \begin{array}{ll}
\left|x_{i+1}-x_{i}\right|-\frac{\Lambda \delta}{-\alpha_{j} \sqrt{l}} \mid \leq \frac{\delta}{l} & \text { if } x_{i}, x_{i+1} \in N_{w_{l}} \cap I_{j}, \alpha_{j}<0, \\
\left|x_{i+1}-x_{i}\right|-\frac{\lambda \delta}{\alpha_{j} \sqrt{l}} \mid \leq \frac{\delta}{l} & \text { if } x_{i}, x_{i+1} \in N_{w_{l}} \cap I_{j}, \alpha_{j}>0 .
\end{array}
$$

iii) There exists $C>0$ such that, for every open interval $G \subset(0,1)$ with $|G| \geq \frac{C}{\sqrt{l}}$ we have that, for $l$ large enough, $N_{w_{l}} \cap G \neq \emptyset$.

In other words, property ii) enstablishes that the distance between points in $N_{w_{l}}$ is prescribed, of order $\frac{1}{\sqrt{l}}$, and depends on the derivative $\alpha_{j}$ of $w$ on $I_{j}$. The errors of order $\frac{\delta}{l}$ are admitted in order to guarantee i). The last condition instead ensures that we cover any interval $I_{j}$ with points in $N_{w_{l}}$ without creating holes of order larger than $\frac{C}{\sqrt{l}}$ between two consecutive intervals.

Let $\varphi:(0,1) \rightarrow\left(0,1+\frac{\delta N_{l}}{l}\right)$ be defined by $\varphi(x):=x+\frac{\delta}{l} \sharp\left\{N_{w_{l}} \cap(0, x)\right\}$, and, with a little abuse of notation, let $\varphi^{-1}:\left(0,1+\frac{\delta N_{l}}{l}\right) \rightarrow(0,1)$ be the function that coincides with the inverse of $\varphi$ on its image, and extended to the whole interval $\left(0,1+\frac{\delta N_{l}}{l}\right)$ so that it is continuous and monotone (such an extension clearly exists and is unique).

Now, we let $P(t):=\left(1-\left(\varphi^{-1}\right)^{\prime}(t)\right)$ for all $t \in\left(0,1+\frac{\delta N_{l}}{l}\right)$, and set $\lambda:(0,1) \rightarrow \mathbb{R}$ to be equal either to $\lambda$ or to $\Lambda$, if $w^{\prime}(x)$ is positive or negative, respectively. Then, we set

$$
\begin{aligned}
\tilde{w}_{l}(x):= & w_{l}\left(\varphi^{-1}(x)\right), \quad \tilde{g}_{l}(x):=w(0)+\int_{0}^{x} P(t) \sqrt{l} \lambda\left(\varphi^{-1}(t)\right) d t, \\
& \text { for all } x \in\left(0,1+\frac{\delta N_{l}}{l}\right) .
\end{aligned}
$$

Now we are in a position to introduce the recovery sequence

$$
g_{l}:=\left(\tilde{w}_{l}+\tilde{g}_{l}\right)\llcorner[0,1] .
$$

By construction $g_{l}$ is admissible; the check is left to the reader. 
First, we show that $\tilde{g}_{l}\left\llcorner(0,1) \rightarrow w\right.$ strongly in $L^{\infty}(0,1)$. More precisely, we shall prove that

$$
\left\|\tilde{g}_{l}-w\right\|_{L^{\infty}(0,1)} \leq \frac{C}{\sqrt{l}}
$$

for some $C \in \mathbb{R}$. To this purpose, it is enough to estimate $\tilde{g}_{l}(x)-w(x)$ only for $x \in I_{1}$, since such an estimate can be clearly iterated for the remaining (finite) intervals. Without loss of generality, we assume $\alpha_{1}>0$. By properties ii) and iii) above we have that, for all $x \in I_{1}$,

$$
\left|\sharp\left\{N_{w_{l}} \cap(0, x)\right\}-x \frac{\alpha_{1} \sqrt{l}}{\lambda \delta}\right| \leq C,
$$

for some constant $C$ independent of $l$. As a consequence, by a change of variables and by its very definition,

$$
\left|\tilde{g}_{l}(\varphi(x))-w(0)-\sharp\left\{N_{w_{l}} \cap(0, x)\right\} \frac{\delta \lambda}{\sqrt{l}}\right| \leq \frac{\delta \lambda}{\sqrt{l}} \quad \text { for all } x \in I_{1} .
$$

Setting $I_{1}=\left[0, p_{1}\right]$, since

$$
\left|\varphi\left(p_{1}\right)-p_{1}\right| \leq \frac{C}{\sqrt{l}}
$$

we deduce that

$$
\left|w(\varphi(x))-w(0)-\sharp\left\{N_{w_{l}} \cap(0, x)\right\} \frac{\delta \lambda}{\sqrt{l}}\right| \leq\left|\alpha_{1}(\varphi(x)-x)\right|+\frac{C}{\sqrt{l}} \leq \frac{C}{\sqrt{l}}
$$

holds for all $x \in \varphi^{-1}\left(I_{1}\right) \subset I_{1}$ and $l$ large enough. Moreover, thanks to (35), we conclude that (36), in fact, holds true on the whole $I_{1}$. This, together with (34) and by triangular inequality yields,

$$
\left|\tilde{g}_{l}(\varphi(x))-w(\varphi(x))\right| \leq \frac{C}{\sqrt{l}} \quad \text { for all } x \in I_{1},
$$

from which (33) easily follows.

Now we prove that

$$
\left\|\tilde{g}_{l}-w\right\|_{H^{\frac{1}{2}(0,1)}} \rightarrow 0 \quad \text { as } \quad l \rightarrow+\infty .
$$

Given $M \in \mathbb{N}$, we set $J_{\frac{M}{l}}(x):=\left(x-\frac{M}{l}, x+\frac{M}{l}\right) \cap[0,1]$ for every $x \in(0,1)$ and define $h_{l}:=\tilde{g}_{l}-w$. Moreover, we set

$$
F_{l}:=\left\{x \in[0,1]: \tilde{g}_{l}^{\prime} \equiv 0 \text { on } J_{\frac{M}{l}}(x)\right\} .
$$

Note that $\left|F_{l}^{c}\right| \leq \frac{C M}{\sqrt{l}}$, since the number of points in $N_{w(l)}$ is of order $\sqrt{l}$. Recalling (33):

$$
\begin{aligned}
\left\|\tilde{g}_{l}-w\right\|_{\dot{H}^{\frac{1}{2}}(0,1)} & =\int_{0}^{1}\left(\int_{J_{\frac{M}{T}}(x)} \frac{\left|h_{l}(x)-h_{l}(y)\right|^{2}}{|x-y|^{2}} d y+\int_{J_{\frac{M}{l}}^{c}(x)} \frac{\left|h_{l}(x)-h_{l}(y)\right|^{2}}{|x-y|^{2}} d y\right) d x \\
& \leq \int_{F_{l}} \int_{J_{\frac{M}{T}}(x)} C d y d x+\int_{F_{l}^{c}} \int_{J_{\frac{M}{T}}(x)} C l d y d x \\
& +\int_{0}^{1} \frac{C}{l} \int_{J_{\frac{M}{T}}^{c}(x)} \frac{1}{|x-y|^{2}} d y d x
\end{aligned}
$$




$$
\begin{aligned}
& \leq \frac{C M}{l}+\left|F_{l}^{c}\right| \frac{2 M}{l} C l+\int_{0}^{1} \frac{C}{M} d x \\
& \leq \frac{C M}{l}+\frac{C M^{2}}{\sqrt{l}}+\int_{0}^{1} \frac{C}{M} d x,
\end{aligned}
$$

where in the first inequality we have used that $w^{\prime}$ is of order 1 , that $\tilde{g}_{l}^{\prime}$ is of order $\sqrt{l}$ on $F_{l}^{c}$, and that $h_{l}$ is of order $\frac{1}{\sqrt{l}}$. Notice that the last term converges to $\frac{C}{M}$ as $l \rightarrow+\infty$. By sending $M \rightarrow+\infty$ and recalling (33) we hence deduce (37).

It remains to show that

$$
\limsup _{l \rightarrow+\infty}\left\|\tilde{w}_{l}\right\|_{\dot{H}^{\frac{1}{2}}(0,1)}^{2} \leq c \text {. }
$$

Indeed, from (39) it follows that $\tilde{w}_{l}$, up to translations, is pre-compact in $H^{\frac{1}{2}}$, and by Proposition 2.5 it converges, still up to translations, to zero in measure. Recalling that $\tilde{w}_{l}$ have zero mean, we deduce that $\tilde{w}_{l} \rightarrow 0$ in $H^{\frac{1}{2}}(0,1)$, that together with (37) and (32) yields (30). Moreover, by (32), (37), and the definition of $c_{\infty}$, we deduce (31) as follows

$$
\begin{aligned}
\limsup _{l \rightarrow+\infty} F^{l}\left(g_{l}\right) & =\limsup _{l \rightarrow+\infty}\left[\left\|\tilde{w}_{l}\right\|_{\dot{H}^{\frac{1}{2}}(0,1)}^{2}+\left\|\tilde{g}_{l}\right\|_{\dot{H}^{\frac{1}{2}}(0,1)}^{2}+2\left\langle\tilde{w}_{l}, \tilde{g}_{l}\right\rangle_{\dot{H}^{\frac{1}{2}}}\right] \\
& \leq \limsup _{l \rightarrow+\infty}\left\|\tilde{w}_{l}\right\|_{\dot{H}^{\frac{1}{2}(0,1)}}^{2}+\limsup _{l \rightarrow+\infty}\left\|\tilde{g}_{l}\right\|_{\dot{H}^{\frac{1}{2}}(0,1)}^{2} \\
& \leq c_{\infty}+\|w\|_{\dot{H}^{\frac{1}{2}(0,1)}}^{2}=F^{\infty}(w) .
\end{aligned}
$$

We will now prove (39). Since $|\varphi(s)-\varphi(t)| \geq|s-t|$ for all $s, t \in(0,1)$, we have

$$
\begin{aligned}
& \left\|\tilde{w}_{l}\right\|_{\dot{H}^{\frac{1}{2}}\left(\left[0,1+\frac{\delta N_{l}}{l}\right]\right)}^{2} \leq \int_{0}^{1} \int_{0}^{1} \frac{\left(w_{l}(s)-w_{l}(t)\right)^{2}}{|s-t|^{2}} d s d t \\
& +\sum_{\substack{x_{i} \neq x_{j} \\
x_{i}, x_{j} \in N_{w_{l}}}} \frac{\delta^{2}}{l^{2}} \frac{\left(w_{l}\left(x_{i}\right)-w_{l}\left(x_{j}\right)\right)^{2}}{\left|x_{i}-x_{j}\right|^{2}} \\
& +\frac{2 \delta}{l} \sum_{x_{i} \in N_{w_{l}}} \int_{0}^{1} \frac{\left(w_{l}\left(x_{i}\right)-w_{l}(t)\right)^{2}}{\left|x_{i}-t\right|^{2}} d t .
\end{aligned}
$$

The first term is uniformly bounded by a constant independent of $l$; Therefore, we have to prove that the terms in (40) tend to 0 as $l \rightarrow+\infty$. To this purpose, for all $x_{i} \in N_{w_{l}}$ we set $I_{i}^{l}:=\left(x_{i}-\frac{\delta}{2 l}, x_{i}+\frac{\delta}{2 l}\right)$, and we denote by $U^{l}$ their union. We have

$$
\begin{aligned}
& \sum_{\substack{x_{i} \neq x_{j} \\
x_{i}, x_{j} \in N_{w_{l}}}} \frac{\delta^{2}}{l^{2}} \frac{\left(w_{l}\left(x_{i}\right)-w_{l}\left(x_{j}\right)\right)^{2}}{\left|x_{i}-x_{j}\right|^{2}} \\
& =\sum_{\substack{x_{i} \neq x_{j} \\
x_{i}, x_{j} \in N_{w_{l}}}} \int_{I_{i}^{l}} \int_{I_{j}^{l}} \frac{\left(w_{l}\left(x_{i}\right)-w_{l}\left(x_{j}\right)\right)^{2}}{\left|x_{i}-x_{j}\right|^{2}} d s d t \\
& \leq 2 \sum_{\substack{x_{i} \neq x_{j} \\
x_{i}, x_{j} \in N_{w_{l}}}} \int_{I_{i}^{l}} \int_{I_{j}^{l}} \frac{\left(w_{l}\left(x_{i}\right)-w_{l}\left(x_{j}\right)\right)^{2}}{|s-t|^{2}} d s d t
\end{aligned}
$$




$$
\begin{aligned}
\leq & C \int_{U^{l}} \int_{U^{l}} \frac{\left(w_{l}(s)-w_{l}(t)\right)^{2}}{|s-t|^{2}} d s d t \\
& +C \sum_{x_{i}, x_{j} \in N_{w_{l}}} \int_{I_{i}^{l}} \int_{I_{j}^{l}} \frac{\left(w_{l}\left(x_{i}\right)-w_{l}(t)\right)^{2}}{|s-t|^{2}} d s d t
\end{aligned}
$$

The term in (41) converges to zero, thanks to Lemma 3.2 and via a change of variables. The term in (42) can be easily estimated first integrating in $s$, exploiting the fact that $w_{l}^{\prime} \leq \Lambda \sqrt{l}$, and property ii), so that

$$
C \sum_{x_{i}, x_{j} \in N_{w_{l}}} \int_{I_{i}^{l}} \int_{I_{j}^{l}} \frac{\left(w_{l}\left(x_{i}\right)-w_{l}(t)\right)^{2}}{|s-t|^{2}} d s d t \leq C\left(\sharp N_{w_{l}}\right)^{2} \frac{\delta^{2}}{l^{2}}\left(\Lambda \sqrt{l} \frac{\delta}{l}\right)^{2} l \leq \frac{C}{l},
$$

which also converges to 0 as $l \rightarrow+\infty$. It remains to estimate the second term in (40); we have

$$
\begin{aligned}
& \frac{2 \delta}{l} \sum_{x_{i} \in N_{w_{l}}} \int_{0}^{1} \frac{\left(w_{l}\left(x_{i}\right)-w_{l}(t)\right)^{2}}{\left|x_{i}-t\right|^{2}} d t=2 \sum_{x_{i} \in N_{w_{l}}} \int_{0}^{1} \int_{I_{i}} \frac{\left(w_{l}\left(x_{i}\right)-w_{l}(t)\right)^{2}}{\left|x_{i}-t\right|^{2}} d s d t \\
& =2 \sum_{x_{i} \in N_{w_{l}}} \int_{I_{i}} \int_{I_{i}} \frac{\left(w_{l}\left(x_{i}\right)-w_{l}(t)\right)^{2}}{\left|x_{i}-t\right|^{2}} d s d t+2 \sum_{x_{i} \in N_{w_{l}}} \int_{I_{i}^{c}} \int_{I_{i}} \frac{\left(w_{l}\left(x_{i}\right)-w_{l}(t)\right)^{2}}{\left|x_{i}-t\right|^{2}} d s d t \\
& =2 \sum_{x_{i} \in N_{w_{l}}} \int_{I_{i}} \int_{I_{i}} \frac{\left(w_{l}\left(x_{i}\right)-w_{l}(t)\right)^{2}}{\left|x_{i}-t\right|^{2}} d s d t \\
& \quad+C \sum_{x_{i} \in N_{w_{l}}}\left[\int_{I_{i}^{c}} \int_{I_{i}} \frac{\left(w_{l}\left(x_{i}\right)-w_{l}(s)\right)^{2}}{\left|x_{i}-t\right|^{2}} d s d t+\int_{I_{i}^{c}} \int_{I_{i}} \frac{\left(w_{l}(s)-w_{l}(t)\right)^{2}}{|s-t|^{2}} d s d t\right] .
\end{aligned}
$$

The last term tends to zero, again thanks to Lemma 3.2 and via a change of variables. Moreover, since $w_{l}^{\prime}$ is bounded by $C \sqrt{l}$,

$$
2 \sum_{x_{i} \in N_{w_{l}}} \int_{I_{i}} \int_{I_{i}} \frac{\left(w_{l}\left(x_{i}\right)-w_{l}(t)\right)^{2}}{\left|x_{i}-t\right|^{2}} d s d t \leq C \sqrt{l} \frac{1}{l^{2}} l
$$

and the right hand side converges to 0 as $l \rightarrow+\infty$. Finally, integrating in $t$ and using again the Lipschitz continuity of $w_{l}$, we get

$$
C \sum_{x_{i} \in N_{w_{l}}} \int_{I_{i}^{c}} \int_{I_{i}} \frac{\left(w_{l}\left(x_{i}\right)-w_{l}(s)\right)^{2}}{\left|x_{i}-t\right|^{2}} d s d t \leq C \sum_{x_{i} \in N_{w_{l}}} \int_{I_{i}} \frac{\frac{C}{l}}{\frac{\delta}{2 l}} d s \leq \frac{C}{\sqrt{l}} .
$$

Casting these estimates in (43), we deduce that also the last term in (40) tends to zero, which in turn yields (39) and concludes the proof of the $\Gamma$-limsup inequality.

\section{Periodicity of dislocations on $\mathcal{S}^{1}$}

\subsection{Admissible configurations and the energy functional}

In order to study the optimal positioning of dislocations we restrict ourselves to the analysis of a simplified model. Roughly speaking, we neglect boundary effects by working on $\mathcal{S}^{1}$; 
then, we will consider the limit of the energy induced by a finite number of dislocations (on $\left.\mathcal{S}^{1}\right)$ as $\Lambda \rightarrow+\infty$. To this purpose, we consider the new distance on $(0,1)$ defined by

$$
d(x, y)=\min \{|x-y|, 1-|x-y|\} .
$$

We fix the number $N \in \mathbb{N}$ of dislocations, and consider families of points $\left(x_{1}, \ldots, x_{N}\right) \in$ $[0,1]^{N}$ which represent the dislocation positions. For convenience we will "cut and paste" the dislocations on the whole $\mathbb{R}$, by setting

$$
\left\{y_{i}\right\}_{i \in I}:=\left\{y \in \mathbb{R}: y=x_{j}+k, 1 \leq j \leq N, k \in \mathbb{Z}\right\} .
$$

Assume now

$$
\delta=\frac{\lambda}{N(\lambda+\Lambda)}
$$

The class of admissible displacements is defined as

$$
\begin{gathered}
\mathcal{V}^{\Lambda}:=\left\{v \in W^{1, \infty}(\mathbb{R}): \exists\left(x_{1}, \ldots, x_{N}\right) \in(0,1)^{N}: d\left(x_{i}, x_{j}\right) \geq \delta \forall i \neq j,\right. \\
\left.v^{\prime}=\lambda-(\lambda+\Lambda) \sum_{i \in I} \chi_{E_{i}}, E_{i}:=\left(y_{i}-\frac{\delta}{2}, y_{i}+\frac{\delta}{2}\right)\right\},
\end{gathered}
$$

where the points $y_{i}$ are defined in (44). Notice that by definition the function $v^{\prime}$ is periodic on $\mathbb{R}$ with period equal to 1 , and that the condition $\delta=\frac{\lambda}{N(\lambda+\Lambda)}$ enforces $v(0)=v(1)$; in this way $v$ is periodic with period equal to 1 and continuous on the whole $\mathbb{R}$. The energy of the system is given by

$$
E(v):=\int_{0}^{1} \int_{0}^{1} \frac{|v(x)-v(y)|^{2}}{d(x-y)^{2}} d x d y
$$

and can be equivalently expressed as in the following Lemma.

Lemma 4.1 For $v \in \mathcal{V}^{\Lambda}$ we have that

$$
E(v)=\int_{0}^{1} \int_{-\frac{1}{2}}^{\frac{1}{2}} \frac{|h(y+z)-h(y)|^{2}}{|z|^{2}} d z d y-\lambda^{2},
$$

where $h(t):=v(t)-\lambda t$.

Proof Since $v$ and $d$ are both 1-periodic, then the energy can be computed as

$$
E(v)=\int_{0}^{1} \int_{y-\frac{1}{2}}^{y+\frac{1}{2}} \frac{|v(x)-v(y)|^{2}}{|x-y|^{2}} d x d y
$$

as $d(x-y)=|x-y|$ on the integration domain. Note that

$$
\frac{|v(x)-v(y)|^{2}}{|x-y|^{2}}=\lambda^{2}+\frac{|h(x)-h(y)|^{2}}{|x-y|^{2}}+2 \lambda \frac{h(x)-h(y)}{x-y} .
$$

Now recall that $v$ is 1 -periodic, so that $h(y+1)=h(y)-\lambda$. Therefore, by introducing the new variable $z:=x-y$ we have 


$$
\begin{aligned}
& \int_{0}^{1} \int_{y-\frac{1}{2}}^{y+\frac{1}{2}} \frac{h(x)-h(y)}{x-y} d x d y=\int_{-\frac{1}{2}}^{\frac{1}{2}} \frac{1}{z}\left(\int_{0}^{1} h(y+z)-h(y) d y\right) d z \\
& =\int_{-\frac{1}{2}}^{\frac{1}{2}} \frac{1}{z}\left(\int_{0}^{z} h(y+1)-h(y) d y\right) d z=-\lambda .
\end{aligned}
$$

Integrating both sides in (49) and again by the change of variable $z:=x-y$, in view of (50) we conclude (48).

In view of the above lemma, we introduce the class

$$
\mathcal{H}^{\Lambda}:=\left\{v-\lambda I d, v \in \mathcal{V}^{\Lambda}\right\} .
$$

Now we are interested in considering the limit as $\Lambda \rightarrow+\infty$ of the proposed model; this, recalling (45), corresponds to sending $\delta \rightarrow 0$. Notice that if $h^{\Lambda} \in \mathcal{H}^{\Lambda}$, then, up to a subsequence and up to additive constants, $h^{\Lambda}$ converges strongly in $L^{1}(0,1)$ (and in fact in all $\left.L^{p}, p<\infty\right)$ and pointwise almost everywhere to a step function $h$, as $\Lambda \rightarrow+\infty$. Therefore (since $h$ is not constant) $\|h\|_{\dot{H}^{\frac{1}{2}}}=+\infty$. To overcome this problem we cut off the core region around dislocation points. Specifically, for fixed $\rho>\frac{\delta}{2}>0$ and $\Lambda>0$ (large enough) we consider the energy functionals $E_{\rho}^{\Lambda}: \mathcal{H}^{\Lambda} \rightarrow[0,+\infty)$ defined by

$$
E_{\rho}^{\Lambda}(h):=\int_{0}^{1} \int_{-\frac{1}{2}}^{-\rho} \frac{|h(y+z)-h(y)|^{2}}{|z|^{2}} d z d y+\int_{0}^{1} \int_{\rho}^{\frac{1}{2}} \frac{|h(y+z)-h(y)|^{2}}{|z|^{2}} d z d y .
$$

Then we study the convergence of the functionals $E_{\rho}^{\Lambda}$ as $\Lambda \rightarrow+\infty$.

Let us set $I_{\rho}:=\left(-\frac{1}{2},-\rho\right) \cup\left(\rho, \frac{1}{2}\right)$. The functionals above read

$$
E_{\rho}^{\Lambda}(h)=\left\|\Delta_{h}\right\|_{L^{2}\left([0,1] \times I_{\rho}\right)}^{2},
$$

where $\Delta_{h}(y, z)=\frac{h(y+z)-h(y)}{z}$. Notice that $h(0)-h(1)=\lambda$ and that the slope of $h$ on $[0,1] \times I_{\rho}$ is less or equal to $\frac{h(y+z)-h(y)}{\rho} \leq \frac{\lambda}{\rho}$. Hence up to adding a suitable constant to $h$ we have

$$
\|h\|_{B V(0,1)} \leq 2 \lambda, \quad\left\|\Delta_{h}(y, z)\right\|_{L^{\infty}\left([0,1] \times I_{\rho}\right)} \leq \frac{\lambda}{\rho} \quad \text { for all } h \in \mathcal{H}^{\Lambda} .
$$

Let now $h^{\Lambda} \in \mathcal{H}^{\Lambda}$; up to subsequences, $h^{\Lambda} \rightarrow h$ strongly in $L^{p}(0,1)$, for all $p<\infty$. Without loss of generality we may assume $h^{\Lambda} \rightarrow h$ a.e. so that $\Delta_{h^{\Lambda}} \rightarrow \Delta_{h}$ a.e. in $[0,1] \times I_{\rho}$. Thanks to the boundedness (52) we infer $\Delta_{h^{\Lambda}} \rightarrow \Delta_{h}$ strongly in $L^{2}$ and we conclude

$$
E_{\rho}^{\Lambda}\left(h^{\Lambda}\right) \rightarrow E_{\rho}(h):=\iint_{[0,1] \times I_{\rho}} \frac{|h(y+z)-h(y)|^{2}}{|z|^{2}} d z d y .
$$

Therefore, the asymptotic behavior of minimizers of $E_{\rho}^{\Lambda}$, as $\Lambda \rightarrow+\infty$, is described by the ground states of the more tractable functionals $E_{\rho}$ defined on step functions.

In order to study the periodicity of minimizers of the energy introduced above, it is convenient to rewrite the energy as a function of the dislocation points. We assume that the dislocations are at a minimal distance $\rho$ with $\frac{1}{N} \geq \rho>0$. Then, we introduce the class of admissible dislocations $\mathcal{A D}_{\rho}^{N}$ defined as

$$
\mathcal{A D}_{\rho}^{N}:=\left\{\left\{x_{1}, \ldots, x_{N}\right\} \subset[0,1): d\left(x_{i}, x_{j}\right) \geq \rho \quad \text { for all } i \neq j\right\} .
$$


Given $X \in \mathcal{A D}_{\rho}^{N}$, we set

$$
Y(X):=\left\{y_{i} \in \mathbb{R}: y_{i}=x_{j}+k, 1 \leq j \leq N, k \in \mathbb{Z}\right\} .
$$

Now, the energy $E_{\rho}$ can be regarded as a function of the dislocation points: We introduce the energy functional $\mathcal{E}_{\rho}^{N}: \mathcal{A D}_{\rho}^{N} \rightarrow \mathbb{R}$ defined by

$$
\mathcal{E}_{\rho}^{N}(X)=E_{\rho}\left(h_{X}\right) \text { for all } X=\left\{x_{1}, \ldots, x_{N}\right\},
$$

where $h_{X} \in B V_{\text {loc }}(\mathbb{R})$ is defined, up to an additive constant, by the condition

$$
h_{X}^{\prime}=-\frac{\lambda}{N} \sum_{y \in Y(X)} \delta_{y},
$$

and $E_{\rho}$ is defined in (53). With a little abuse of notation, given $X=\left\{x_{1}, \ldots, x_{N}\right\} \in \mathcal{A D}_{\rho}^{N}$ we will also write $\mathcal{E}_{\rho}^{N}\left(x_{1}, \ldots, x_{N}\right)=\mathcal{E}_{\rho}^{N}(X)$.

The following theorem establishes that the energy $\mathcal{E}_{\rho}^{N}$ is minimized on configuration of equi-spaced dislocations.

Theorem 4.2 Let $0<\rho<\frac{1}{N}$. The energy $\mathcal{E}_{\rho}^{N}$ at (54) admits a minimizer $X \in \mathcal{A D}_{\rho}^{N}$; moreover each minimizer is of the form $X=\left\{x_{1}, \ldots, x_{N}\right\}$, where $x_{1}<\cdots<x_{N}$ and $d\left(x_{i}, x_{i+1}\right)=\frac{1}{N}$ for every $i=1, \ldots, N-1$.

\subsection{Proof of Theorem 4.2}

In order to prove Theorem 4.2, we first provide an equivalent formulation for the energy $\mathcal{E}_{\rho}^{N}$ at (54), and subsequently exploit its convexity. Instead of manipulating $\mathcal{E}_{\rho}^{N}$ directly, which seems to be an involved and tedious task, we start by computing its first variation in Step 1. After that, we show how the first variation of $\mathcal{E}_{\rho}^{N}$ coincides with the one of a new functional $\tilde{\mathcal{E}}_{\rho}^{N}$, which is easier to manipulate. Finally, in Step 3, we show that $\tilde{\mathcal{E}}_{\rho}^{N}$ is minimized when the points $x_{1}, \ldots, x_{N}$ are evenly spaced on $\mathcal{S}^{1}$ : This fact is deduced after noting that $\tilde{\mathcal{E}}_{\rho}^{N}$ is a convex function of the $\mathcal{S}^{1}$-distances between points $x_{i}$.

Step 1. Computing the first variation. Since $\lambda$ is fixed we assume for simplicity of notation that $\frac{\lambda}{N}=1$. Let us fix a configuration $X=\left\{x_{1}, \ldots, x_{N}\right\} \in \mathcal{A D}_{\rho}^{N}$, such that $d\left(x_{i}, x_{j}\right)>\rho$ for each $i \neq j$. Fix $i$ and consider the first variation of the energy

$$
\lim _{\varepsilon \rightarrow 0} \frac{1}{\varepsilon}\left(\mathcal{E}_{\rho}^{N}\left(x_{1}^{\varepsilon}, \ldots, x_{N}^{\varepsilon}\right)-\mathcal{E}_{\rho}^{N}\left(x_{1}, \ldots, x_{N}\right)\right)
$$

where

$$
x_{j}^{\varepsilon}:= \begin{cases}x_{j} & \text { if } j \neq i, \\ x_{i}+\varepsilon & \text { if } j=i .\end{cases}
$$

In order to compute the limit in (55) we introduce the function $h^{\varepsilon}$ defined, up to additive constants, by $\left(h^{\varepsilon}\right)^{\prime}:=-\sum_{i} \delta_{x_{i}^{\varepsilon}}$. Let us restrict our analysis to the case $\varepsilon>0$, the other case is similar and will yield the same result. Set $h:=h_{X}$ and notice that

$$
h^{\varepsilon}-h=D^{\varepsilon}:=\chi\left(x_{i}, x_{i}+\varepsilon\right) \text {. }
$$

Therefore we can write

$$
\mathcal{E}_{\rho}^{N}\left(x_{1}^{\varepsilon}, \ldots, x_{N}^{\varepsilon}\right)-\mathcal{E}_{\rho}^{N}\left(x_{1}, \ldots, x_{N}\right)
$$




$$
\begin{aligned}
& =\int_{0}^{1} \int_{I_{\rho}} \frac{\left|h^{\varepsilon}(x+z)-h^{\varepsilon}(x)\right|^{2}-|h(x+z)-h(x)|^{2}}{|z|^{2}} d z d x \\
& =\int_{0}^{1} \int_{I_{\rho}} \frac{\left(S^{\varepsilon}(x+z)-S^{\varepsilon}(x)\right)\left(D^{\varepsilon}(x+z)-D^{\varepsilon}(x)\right)}{|z|^{2}} d z d x,
\end{aligned}
$$

where we have set $S^{\varepsilon}(t)=h(t)+h^{\varepsilon}(t)$, while $D^{\varepsilon}$ is defined in (57). For $\varepsilon$ small enough, we have

$$
D^{\varepsilon}(x+z)-D^{\varepsilon}(x)= \begin{cases}-1 & \text { if } x \in\left(x_{i}, x_{i}+\varepsilon\right) \\ 1 & \text { if } x+z \in\left(x_{i}, x_{i}+\varepsilon\right) \\ 0 & \text { otherwise }\end{cases}
$$

Hence, by (57) and (58) we get

$$
\begin{aligned}
& \mathcal{E}_{\rho}^{N}\left(x_{1}^{\varepsilon}, \ldots, x_{N}^{\varepsilon}\right)-\mathcal{E}_{\rho}^{N}\left(x_{1}, \ldots, x_{N}\right) \\
& =\int_{I_{\rho}} \frac{1}{|z|^{2}} \int_{x_{i}-z}^{x_{i}-z+\varepsilon}\left(S^{\varepsilon}(x+z)-S^{\varepsilon}(x)\right) d x-\int_{x_{i}}^{x_{i}+\varepsilon}\left(S^{\varepsilon}(x+z)-S^{\varepsilon}(x)\right) d x d z \\
& =\int_{I_{\rho}} \frac{1}{|z|^{2}} \int_{x_{i}}^{x_{i}+\varepsilon}\left(S^{\varepsilon}(x)-S^{\varepsilon}(x-z)\right)-\left(S^{\varepsilon}(x+z)-S^{\varepsilon}(x)\right) d x d z \\
& =2 \int_{\rho}^{\frac{1}{2}} \frac{1}{|z|^{2}} \int_{x_{i}}^{x_{i}+\varepsilon}\left(S^{\varepsilon}(x)-S^{\varepsilon}(x-z)\right)-\left(S^{\varepsilon}(x+z)-S^{\varepsilon}(x)\right) d x d z \\
& =-2 \int_{I_{\rho}} \frac{1}{|z|^{2}} \int_{x_{i}}^{x_{i}+\varepsilon}\left(S^{\varepsilon}(x+z)-S^{\varepsilon}(x)\right) d x d z .
\end{aligned}
$$

With the aid of an integration by parts, the previous expression equals

$$
\begin{aligned}
& -2 \int_{x_{i}}^{x_{i}+\varepsilon} \frac{S^{\varepsilon}(x+\rho)-S^{\varepsilon}(x)}{\rho}+\frac{S^{\varepsilon}(x-\rho)-S^{\varepsilon}(x)}{\rho} d x \\
& \quad+4 \int_{x_{i}}^{x_{i}+\varepsilon} S^{\varepsilon}\left(x+\frac{1}{2}\right)+S^{\varepsilon}\left(x-\frac{1}{2}\right)-2 S^{\varepsilon}(x) d x \\
& \quad-2 \int_{I_{\rho}} \int_{x_{i}}^{x_{i}+\varepsilon} \frac{\dot{S}^{\varepsilon}(x+z)}{z} d x d z .
\end{aligned}
$$

Exploiting the fact that the $\mathcal{S}^{1}$-distance between the points $x_{j}$ is larger than $\rho$, we easily see that, for $\varepsilon$ small enough, $S^{\varepsilon}(x+\rho)-S^{\varepsilon}(x)=-1$ and $S^{\varepsilon}(x-\rho)-S^{\varepsilon}(x)=1$ for $x \in\left(x_{i}, x_{i}+\varepsilon\right)$, so that the first line is null.

As for the second line, we will compute it as $\varepsilon$ is small; we first see that the values of $S^{\varepsilon}\left(x-\frac{1}{2}\right)$ and $S^{\varepsilon}\left(x+\frac{1}{2}\right)$ do not depend on $\varepsilon$ and equal $2 h\left(x-\frac{1}{2}\right)$ and $2 h\left(x+\frac{1}{2}\right)$, respectively. Moreover $S^{\varepsilon}(x)$ is constant on $\left(x_{i}, x_{i}+\varepsilon\right)$ and coincides with $2 h(x)+1=2 h^{+}\left(x_{i}\right)+1$ (where $h^{+}(t)=\lim _{s \rightarrow t^{+}} h(s)$ ), so that

$$
\begin{gathered}
\lim _{\varepsilon \rightarrow 0} \frac{1}{\varepsilon} \int_{x_{i}}^{x_{i}+\varepsilon} S^{\varepsilon}\left(x+\frac{1}{2}\right)+S^{\varepsilon}\left(x-\frac{1}{2}\right)-2 S^{\varepsilon}(x) d x \\
=2 h^{+}\left(x_{i}-\frac{1}{2}\right)+2 h^{+}\left(x_{i}+\frac{1}{2}\right)-4 h^{+}\left(x_{i}\right)-2 .
\end{gathered}
$$


We write $2 h^{+}\left(x_{i}\right)+1=h^{+}\left(x_{i}\right)+h^{-}\left(x_{i}\right)$, so that

$$
\begin{aligned}
& 2 h^{+}\left(x_{i}-\frac{1}{2}\right)+2 h^{+}\left(x_{i}+\frac{1}{2}\right)-4 h^{+}\left(x_{i}\right)-2 \\
& \quad=2\left(h^{+}\left(x_{i}-\frac{1}{2}\right)-h^{-}\left(x_{i}\right)\right)+2\left(h^{+}\left(x_{i}+\frac{1}{2}\right)-h^{+}\left(x_{i}\right)\right),
\end{aligned}
$$

and we observe that

$$
\begin{aligned}
& h^{+}\left(x_{i}-\frac{1}{2}\right)-h^{-}\left(x_{i}\right)=\sharp\left(Y(X) \cap\left(x_{i}-\frac{1}{2}, x_{i}\right)\right), \\
& h^{+}\left(x_{i}+\frac{1}{2}\right)-h^{+}\left(x_{i}\right)=-\sharp\left(Y(X) \cap\left(x_{i}, x_{i}+\frac{1}{2}\right]\right) .
\end{aligned}
$$

By (62), (63) and (64) we conclude that

$$
\lim _{\varepsilon \rightarrow 0} \frac{4}{\varepsilon} \int_{x_{i}}^{x_{i}+\varepsilon} S^{\varepsilon}\left(x+\frac{1}{2}\right)+S^{\varepsilon}\left(x-\frac{1}{2}\right)-2 S^{\varepsilon}(x) d x=-8 \Delta\left(x_{i}\right),
$$

where we have set

$$
\Delta\left(x_{i}\right):=\sharp\left(Y(X) \cap\left(x_{i}, x_{i}+\frac{1}{2}\right]\right)-\sharp\left(Y(X) \cap\left(x_{i}-\frac{1}{2}, x_{i}\right)\right) .
$$

Let us finally analyse the last line in (61). To do this we first recall that

$$
\dot{S}^{\varepsilon}=\left(-2 \sum_{y \in Y(X), y \neq x_{i}} \delta_{y}\right)-\delta_{x_{i}+\varepsilon}-\delta_{x_{i}},
$$

which yields, for a.e. $x \in\left(x_{i}, x_{i}+\varepsilon\right)$,

$$
\int_{x+\rho}^{x+\frac{1}{2}} \frac{\dot{S}^{\varepsilon}(z)}{z-x} d z+\int_{x-\frac{1}{2}}^{x-\rho} \frac{\dot{S}^{\varepsilon}(z)}{z-x} d z=-2 \sum_{\substack{y \in\left(x+\rho, x+\frac{1}{2}\right] \\ y \in Y(X)}} \frac{1}{y-x}-2 \sum_{\substack{y \in\left(x-\frac{1}{2}, x-\rho\right) \\ y \in Y(X)}} \frac{1}{y-x} .
$$

Notice that in the above sum, the terms containing $x_{i}+\varepsilon$ and $x_{i}$ do not appear because $x \in\left(x_{i}, x_{i}+\varepsilon\right)$. By integrating (67) with respect to $x \in\left(x_{i}, x_{i}+\varepsilon\right)$, we get that, for $\varepsilon$ small enough, the last line in (61) equals

$$
\begin{gathered}
4 \int_{x_{i}}^{x_{i}+\varepsilon} \sum_{\substack{y \in\left(x+\rho, x+\frac{1}{2}\right] \\
y \in Y(X)}} \frac{1}{y-x}+\sum_{\substack{y \in\left(x-\frac{1}{2}, x-\rho\right) \\
y \in Y(X)}} \frac{1}{y-x} d x \\
=-4 \sum_{\substack{y \in\left(x_{i}+\rho, x_{i}+\frac{1}{2}\right] \\
y \in Y(X)}} \log \left(\left|y-x_{i}-\varepsilon\right|\right)-\log \left(\left|y-x_{i}\right|\right) \\
-4 \sum_{\substack{y \in\left(x_{i}-\frac{1}{2}, x_{i}-\rho\right) \\
y \in Y(X)}} \log \left(\left|y-x_{i}-\varepsilon\right|\right)-\log \left(\left|y-x_{i}\right|\right) .
\end{gathered}
$$

Putting together (60), (61), (65), (68) we infer

$$
\lim _{\varepsilon \rightarrow 0^{+}} \frac{1}{\varepsilon}\left(\mathcal{E}_{\rho}^{N}\left(x_{1}^{\varepsilon}, \ldots, x_{N}^{\varepsilon}\right)-\mathcal{E}_{\rho}^{N}\left(x_{1}, \ldots, x_{N}\right)\right)
$$




$$
=-8 \Delta\left(x_{i}\right)-4 \sum_{\substack{y \in\left(x_{i}-\frac{1}{2}, x_{i}\right) \\ y \in Y(X)}} \frac{1}{\left|y-x_{i}\right|}+4 \sum_{\substack{y \in\left(x_{i}, x_{i}+\frac{1}{2}\right] \\ y \in Y(X)}} \frac{1}{\left|y-x_{i}\right|} .
$$

where we have also used that $Y(X) \cap\left(x_{i}+\rho, x_{i}+\frac{1}{2}\right]=Y(X) \cap\left(x_{i}, x_{i}+\frac{1}{2}\right]$ and also $Y(X) \cap\left(x_{i}-\frac{1}{2}, x_{i}-\rho\right)=Y(X) \cap\left(x_{i}-\frac{1}{2}, x_{i}\right)$.

As anticipated, the computation in the case $\varepsilon<0$ is similar and yields the same limit, hence proving that the quantity above is the first variation of the energy $\mathcal{E}_{\rho}^{N}$.

Notice that

$$
\Delta\left(x_{i}\right)=\sum_{\substack{y \in\left(x_{i}, x_{i}+\frac{1}{2}\right] \\ y \in Y(X)}} \frac{y-x_{i}}{\left|y-x_{i}\right|}+\sum_{\substack{y \in\left(x_{i}-\frac{1}{2}, x_{i}\right) \\ y \in Y(X)}} \frac{y-x_{i}}{\left|y-x_{i}\right|} .
$$

We get

$$
\begin{aligned}
\lim _{\varepsilon \rightarrow 0} \frac{1}{\varepsilon} & \left(\mathcal{E}_{\rho}^{N}\left(x_{1}^{\varepsilon}, \ldots, x_{N}^{\varepsilon}\right)-\mathcal{E}_{\rho}^{N}\left(x_{1}, \ldots, x_{N}\right)\right) \\
& =4\left(\sum_{\substack{y \in\left(x_{i}-\frac{1}{2}, x_{i}\right) \\
y \in Y(X)}} \frac{-1-2\left(y-x_{i}\right)}{\left|y-x_{i}\right|}+\sum_{\substack{y \in\left(x_{i}, x_{i}+\frac{1}{2}\right] \\
y \in Y(X)}} \frac{1-2\left(y-x_{i}\right)}{\left|y-x_{i}\right|}\right) .
\end{aligned}
$$

Step 2. Rewriting $\mathcal{E}_{\rho}^{N}$. It is easy to check that the quantity at (70) coincides with the partial derivative with respect to $x_{i}$ of the functional

$$
\tilde{\mathcal{E}}_{\rho}^{N}\left(x_{1}, \ldots, x_{N}\right):=2 \sum_{i=1}^{N}\left(\sum_{\substack{y \in\left(x_{i}-\frac{1}{2}, x_{i}+\frac{1}{2}\right] \\ y \neq x_{i}, y \in Y(X)}}-\log \left(\left|y-x_{i}\right|\right)+2\left|y-x_{i}\right|\right) .
$$

Notice that if $y \in\left(x_{i}-\frac{1}{2}, x_{i}+\frac{1}{2}\right]$, then $y=x_{j}+k$ for some $x_{j} \in X$ and $k \in\{-1,0,1\}$, and $\left|y-x_{i}\right|=\left|x_{j}-x_{i}\right| \wedge\left(1-\left|x_{j}-x_{i}\right|\right)$. Then, we can also write the functional $\tilde{\mathcal{E}}_{\rho}^{N}$ in the equivalent way

$$
\begin{aligned}
\tilde{\mathcal{E}}_{\rho}^{N}\left(x_{1}, \ldots, x_{N}\right)= & 2 \sum_{i \neq j}\left(-\log \left(\left|x_{j}-x_{i}\right|\right) \vee\left(-\log \left(1-\left|x_{j}-x_{i}\right|\right)\right)\right. \\
& +4 \sum_{i \neq j}\left|x_{j}-x_{i}\right| \wedge\left(1-\left|x_{j}-x_{i}\right|\right) .
\end{aligned}
$$

Finally, we will also make use of the following formula

$$
\tilde{\mathcal{E}}_{\rho}^{N}\left(x_{1}, \ldots, x_{N}\right)=2 \sum_{k=1}^{N-1} G_{k}\left(x_{1}, \ldots, x_{N}\right),
$$

where

$$
\begin{aligned}
G_{k}\left(x_{1}, \ldots, x_{N}\right):= & \sum_{|i-j| \equiv k(\bmod N)}\left(\left(-\log \left(\left|x_{j}-x_{i}\right|\right) \vee\left(-\log \left(1-\left|x_{j}-x_{i}\right|\right)\right)\right)\right. \\
& +2\left(\left|x_{j}-x_{i}\right| \wedge\left(1-\left|x_{j}-x_{i}\right|\right)\right) .
\end{aligned}
$$

Step 3. Minimization. Here we prove that $\tilde{\mathcal{E}}_{\rho}^{N}$ is minimized when $x_{1}, \ldots, x_{N}$ are evenly spaced on $\mathcal{S}^{1}$. To this purpose, we prove that such configurations are the (unique) minimizers 
of $G_{k}$, for each $k=1, \ldots, N-1$. First, we observe that the function

$$
(0,1) \ni y \mapsto f(y):=((-\log (|y|) \vee(-\log (1-|y|)))+2(|y| \wedge(1-|y|))
$$

is strictly convex.

Now, without loss of generality we assume $x_{1}<x_{2}<\cdots<x_{N}$. Then, we fix $k \in$ $\{1, \ldots, N-1\}$ and for all $i=1, \ldots, N$ we set

$$
d_{i}:= \begin{cases}\left|x_{i}-x_{i+k}\right| & \text { if } i+k \leq N \\ 1-\left|x_{i}-x_{i+k-N}\right| & \text { if } i+k>N\end{cases}
$$

so that it turns out that

$$
\sum_{i=1}^{N} d_{i}=k, \quad G_{k}\left(x_{1}, \ldots, x_{N}\right)=\sum_{i=1}^{N} f\left(d_{i}\right) .
$$

Therefore, by Jensen inequality we deduce that $G_{k}$ is minimized if and only if $d_{i}=\frac{k}{N}$ for all $i=1, \ldots, N$. This is achieved if and only if $\left\{x_{i}\right\}_{i=1}^{N}$ are evenly spaced (with respect to the distance $d$ on $\mathcal{S}^{1}$ ). The proof is achieved.

Acknowledgements Open access funding provided by University of Graz. Silvio Fanzon gratefully acknowledges support by the Christian Doppler Research Association (CDG) and Austrian Science Fund (FWF) through the Partnership in Research project PIR-27 "Mathematical methods for motion-aware medical imaging". We authors are members of Gruppo Nazionale per l'Analisi Matematica, la Probabilità e le loro Applicazioni (GNAMPA) of the Istituto Nazionale di Alta Matematica (INDAM). We thank the anonymous referee for the useful suggestions.

Open Access This article is licensed under a Creative Commons Attribution 4.0 International License, which permits use, sharing, adaptation, distribution and reproduction in any medium or format, as long as you give appropriate credit to the original author(s) and the source, provide a link to the Creative Commons licence, and indicate if changes were made. The images or other third party material in this article are included in the article's Creative Commons licence, unless indicated otherwise in a credit line to the material. If material is not included in the article's Creative Commons licence and your intended use is not permitted by statutory regulation or exceeds the permitted use, you will need to obtain permission directly from the copyright holder. To view a copy of this licence, visit http://creativecommons.org/licenses/by/4.0/.

\section{References}

1. Alberti, G., Bouchitté, G., Seppecher, P.: Phase transition with the line-tension effect. Arch. Rational Mech. Anal. 144, 1-46 (1998)

2. Alicandro, R., Lazzaroni, G., Palombaro, M.: On the effect of interactions beyond nearest neighbours on non-convex lattice systems. Calc. Var. Partial Differ. Equ. 56, 42 (2017)

3. Alicandro, R., Lazzaroni, G., Palombaro, M.: Derivation of a rod theory from lattice systems with interactions beyond nearest neighbours. Netw. Heterog. Media 13, 1-26 (2018)

4. Ariza, M., Ortiz, M.: Discrete crystal elasticity and discrete dislocations in crystals. Arch. Rational Mech. Anal. 178(2), 149-226 (2005)

5. Dipierro, S., Palatucci, G., Valdinoci, E.: Dislocation dynamics in crystals: a macroscopic theory in a fractional laplace setting. Commun. Math. Phys. 333, 1061-1105 (2015)

6. Fanzon, S., Palombaro, M., Ponsiglione, M.: A variational model for dislocations at semi-coherent interfaces. J. Nonlinear Sci. 27(5), 1436-1461 (2017)

7. Fanzon, S., Palombaro, M., Ponsiglione, M.: Derivation of linearised polycrystals from a two-dimensional system of edge dislocations. SIAM J. Math. Anal. 51(5), 3956-3981 (2019)

8. Focardi, M., Garroni, A.: A $1 D$ macroscopic phase field model for dislocations and a second order $\Gamma$-limit. SIAM Multiscale Model. Simul. 6(4), 1098-1124 (2007) 
9. Fonseca, I., Fusco, N., Leoni, G., Morini, M.: A model for dislocations in epitaxially strained elastic films. J. Math. Pures Appl. 111(9), 126-160 (2018)

10. Friedrich, M., Stefanelli., U.: Crystallization in a One-Dimensional Periodic Landscape. J. Stat. Phys. 179, 485-501 (2020)

11. Garroni, A., Müller, S.: $\Gamma$-limit of a phase-field model of dislocations. SIAM J. Math. Anal. 36(6), 1943-1964 (2005)

12. Garroni, A., Müller, S.: A model for dislocations in epitaxially strained elastic films. Arch. Rational Mech. Anal. 181(3), 535-578 (2006)

13. Giuliani, A., Theil, F.: Long range order in atomistic models for solids. arXiv e-prints arXiv:1907.07923 (2019)

14. González, M., Monneau, R.: Slow motion of particle systems as a limit of a reaction-diffusion equation with half-laplacian in dimension one. Discrete Contin. Dyn. Syst. 32, 1255-1286 (2012)

15. Hirsch, P.: Nucleation and propagation of misfits dislocations in strained epitaxial layer systems. In Proceedings of the Second International Conference Schwäbisch Hall, Fed. Rep. of Germany, July 30August 31990

16. Kreutz, L., Piovano, P.: Microscopic validation of a variational model of epitaxially strained crystalline film. arXiv e-prints arXiv:1902.06561 (2019)

17. Lauteri, G., Luckhaus, S.: An energy estimate for dislocation configurations and the emergence of cosserattype structures in metal plasticity. arXiv e-prints arXiv:1608.06155 (2016)

18. Lazzaroni, G., Palombaro, M., Schlömerkemper, A.: Rigidity of three-dimensional lattices and dimension reduction in heterogeneous nanowires. Discrete Contin. Dyn. Syst. Ser. S 10, 119-139 (2017)

19. Mironescu, P., Pisante, A.: A variational problem with lack of compactness for $H^{1 / 2}\left(S^{1} ; S^{1}\right)$ maps of prescribed degree. J. Funct. Anal. 217, 249-279 (2004)

20. Müller, S.: Singular perturbations as a selection criterion for periodic minimizing sequences. Calc. Var. Partial Differ. Equ. 1, 169-204 (1993)

21. Müller, S., Palombaro, M.: Derivation of a rod theory for biphase materials with dislocations at the interface. Calc. Var. Partial Differ. Equ. 48(3-4), 315-335 (2013)

22. Nabarro, F.R.N.: Dislocations in a simple cubic lattice. Proc. Phys. Soc. 59, 256-272 (1947)

23. Nabarro, F.R.N.: Theory of Crystal Dislocations. Clarendon Press, Oxford (1967)

24. Ohta, T., Kawasaki, K.: Equilibrium morphology of block copolymer melts. Macromolecules 19, 26212632 (1986)

25. Patrizi, S., Valdinoci, E.: Crystal dislocations with different orientations and collisions. Arch. Rational Mech. Anal. 217, 231-261 (2015)

26. Peierls, R.: The size of a dislocation. Proc. Phys. Soc. 52, 34-37 (1940)

27. Read, W.T., Shockley, W.: Dislocation models of crystal grain boundaries. Phys. Rev. 78, 275-289 (1950)

28. Van Der Merwe, J.: On the stresses and energies associated with inter-crystalline boundaries. Proc. Phys. Soc. A 63, 616-637 (1950)

Publisher's Note Springer Nature remains neutral with regard to jurisdictional claims in published maps and institutional affiliations. 